OPEN ACCESS

Edited by:

Christian Gonzalez-Billault,

University of Chile, Chile

Reviewed by:

Violaine Plante-Bordeneuve,

Hôpitaux Universitaires Henri

Mondor, France

Yifat Miller,

Ben-Gurion University of the

Negev, Israel

*Correspondence:

Maria Rosário Almeida

ralmeida@ibmc.up.pt

Received: 07 August 2020 Accepted: 18 November 2020 Published: 11 December 2020

Citation:

Bezerra F, Saraiva MJ and Almeida MR (2020) Modulation of the Mechanisms Driving Transthyretin

Amyloidosis.

Front. Mol. Neurosci. 13:592644. doi: 10.3389/fnmol.2020.592644

\section{Modulation of the Mechanisms Driving Transthyretin Amyloidosis}

\author{
Filipa Bezerra ${ }^{1,2}$, Maria João Saraiva ${ }^{1,2}$ and Maria Rosário Almeida ${ }^{1,2 \star}$ \\ ${ }^{1}$ Molecular Neurobiology Group, IBMC - Instituto de Biologia Molecular e Celular, i3S - Instituto de Investigação e Inovação \\ em Saúde, Porto, Portugal, ${ }^{2}$ Department of Molecular Biology, ICBAS - Instituto de Ciências Biomédicas Abel Salazar, \\ Universidade do Porto, Porto, Portugal
}

Transthyretin (TTR) amyloidoses are systemic diseases associated with TTR aggregation and extracellular deposition in tissues as amyloid. The most frequent and severe forms of the disease are hereditary and associated with amino acid substitutions in the protein due to single point mutations in the TTR gene (ATTRV amyloidosis). However, the wild type TTR (TTR wt) has an intrinsic amyloidogenic potential that, in particular altered physiologic conditions and aging, leads to TTR aggregation in people over 80 years old being responsible for the non-hereditary ATTRwt amyloidosis. In normal physiologic conditions TTR wt occurs as a tetramer of identical subunits forming a central hydrophobic channel where small molecules can bind as is the case of the natural ligand thyroxine $\left(T_{4}\right)$. However, the TTR amyloidogenic variants present decreased stability, and in particular conditions, dissociate into partially misfolded monomers that aggregate and polymerize as amyloid fibrils. Therefore, therapeutic strategies for these amyloidoses may target different steps in the disease process such as decrease of variant TTR (TTRv) in plasma, stabilization of TTR, inhibition of TTR aggregation and polymerization or disruption of the preformed fibrils. While strategies aiming decrease of the mutated TTR involve mainly genetic approaches, either by liver transplant or the more recent technologies using specific oligonucleotides or silencing RNA, the other steps of the amyloidogenic cascade might be impaired by pharmacologic compounds, namely, TTR stabilizers, inhibitors of aggregation and amyloid disruptors. Modulation of different steps involved in the mechanism of ATTR amyloidosis and compounds proposed as pharmacologic agents to treat TTR amyloidosis will be reviewed and discussed.

Keywords: transthyretin, amyloidosis, mechanism of disease, therapy, amyloid inhibitors

\section{INTRODUCTION}

Amyloidosis comprises a group of diseases which are characterized by extracellular deposition of protein aggregates, with a structure mainly composed of cross $\beta$-sheets, insoluble and toxic, in a range of tissues leading to the dysfunction of normal surrounding tissue (Galant et al., 2017). This review summarizes the current knowledge concerning TTR amyloidosis (ATTR amyloidosis) modulation aiming therapy and discusses the influence of other processes and factors such proteolysis and extracellular chaperones, respectively, on TTR amyloidogenesis in order to contribute for better understanding the disease pathophysiology and for the development of new therapeutic approaches. 


\section{TRANSTHYRETIN (TTR) STRUCTURE AND FUNCTION}

Transthyretin (TTR) is a $55 \mathrm{kDa}$ homotetrameric globular protein constituted by four monomers of 127 amino acid residues (Kanda et al., 1974). It is mainly produced by the liver and choroid plexus of the brain, being then secreted into the blood and cerebrospinal fluid (CSF), respectively. However, TTR synthesis has also been described in other tissues, such as the retinal pigment epithelia (RPE), a monolayer of cells acting as a blood barrier for the retina, which in turn secretes TTR to the vitreous humor (Richardson, 2009). Low levels of TTR expression were also found in Schwann cells of the sciatic nerve, as described by Murakami et al. (2010).

TTR structure was firstly determined in the seventies by Blake and collaborators (Blake et al., 1978), who described that each TTR monomer is organized into two four-stranded anti-parallel $\beta$-sheets (A through $\mathrm{H}$ ) and a short $\beta$-helix located on $\beta$-strand E (Blake et al., 1978); two monomers are connected through hydrogen bonds between the two $\mathrm{H}$ strands of neighboring monomers resulting in a very stable dimer. The association of two dimers, mainly through hydrophobic interactions between residues of the $\mathrm{AB}$ to $\mathrm{GH}$ loops results in the formation of the TTR tetramer (Blake et al., 1978; Yokoyama et al., 2012).

TTR mainly functions as a carrier protein (Buxbaum and Reixach, 2009; Vieira and Saraiva, 2014). The homotetrameric structure of native TTR forms a central hydrophobic channel that harbors two thyroxine $\left(\mathrm{T}_{4}\right)$ binding sites at the dimer-dimer interface (Blake et al., 1974) (Figure 1). However, due to negative cooperativity, only one molecule of $\mathrm{T}_{4}$ is transported by TTR (Andrea et al., 1980). In humans, around $15 \%$ of plasma $\mathrm{T}_{4}$ is transported by TTR, whereas in rodents this percentage increases to $50 \%$ (Vieira and Saraiva, 2014). In the CSF, TTR is the major carrier of $\mathrm{T}_{4}$, transporting around $80 \%$ of the hormone in both humans and rodents (Hu et al., 2006), being recently described as essential for the retention of $\mathrm{T}_{4}$ in the CSF (Chen et al., 2016).

The TTR tetramer has four additional binding sites at the protein's surface for retinol-binding protein (RBP), two in each dimer. Due to steric hindrance, only two RBP molecules may effectively bind to TTR but, since the RBP levels in plasma are lower than TTR, only one RBP molecule is effectively bound to the TTR tetramer (Folli et al., 2010). The assembly of this TTRRBP complex is essential for the transport of retinol (or vitamin A), allowing its delivery to the cells (Raghu and Sivakumar, 2004). Indeed, studies in TTR knockout mice revealed a decrease in both retinol and RBP levels in plasma (van Bennekum et al., 2001), as well as an accumulation of hepatic RBP (Wei et al., 1995), comparatively to wild-type mice. Altogether these results suggest the pivotal role of TTR as a carrier of the retinolRBP complex preventing its glomerular filtration by the kidney (Wei et al., 1995; van Bennekum et al., 2001; Gaetani et al., 2002).

Besides its functions as carrier protein, a proteolytic activity has been attributed to TTR. A small fraction of plasma TTR (1-2\%) was found associated with high density lipoproteins (HDL) via apolipoprotein AI (apoA-I) (Sousa et al., 2000) and,

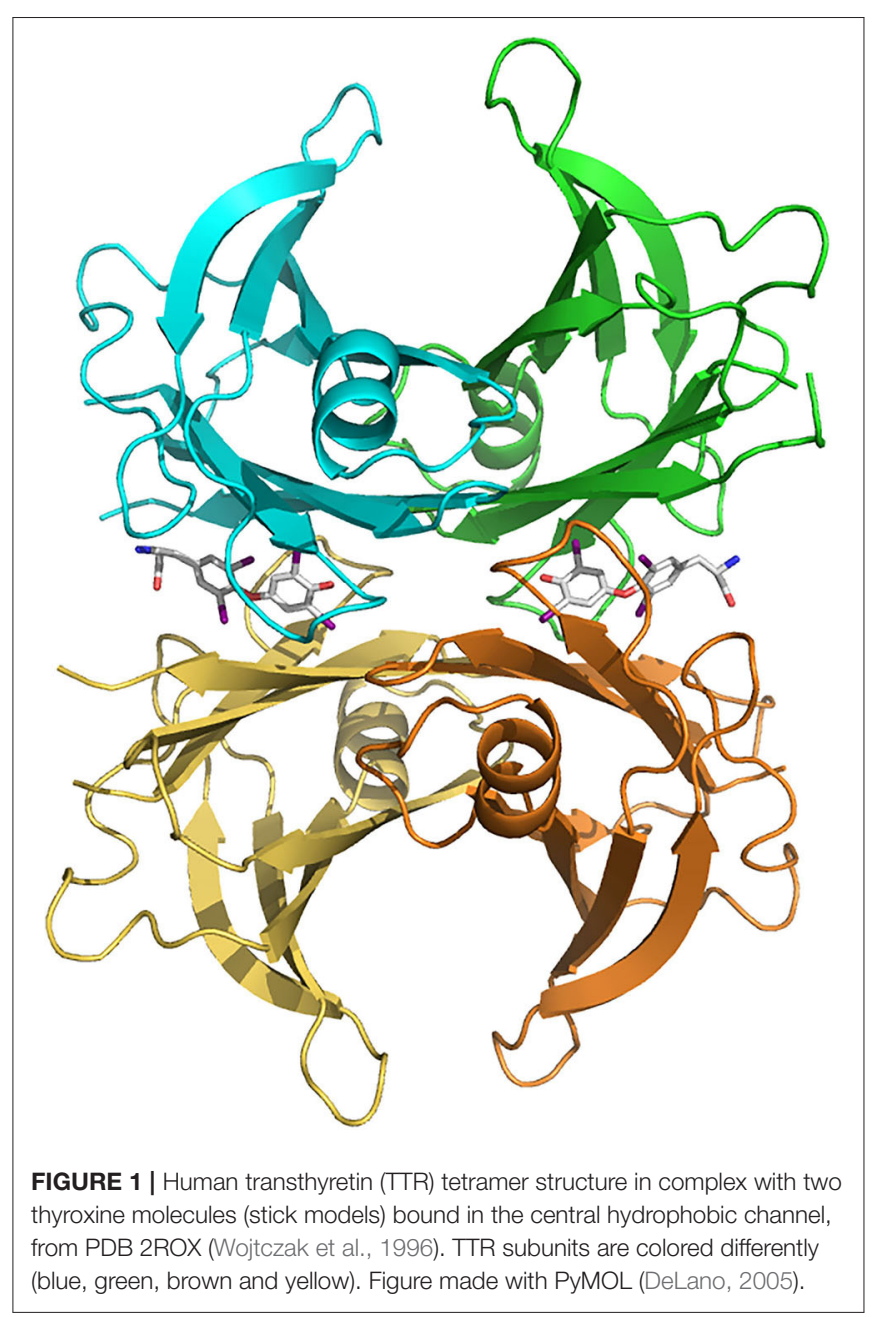

later, the capacity of TTR to cleave apoA-I carboxyl terminal domain in vitro was also demonstrated (Liz et al., 2004). In addition to apoA-I, TTR is also involved on the cleavage of both neuropeptide Y (NPY) (Liz et al., 2009) and A $\beta$ peptide (Costa et al., 2008), suggesting an important role of TTR-mediated proteolysis either in physiologic or pathologic conditions, with major impacts on the biology of nervous system and Alzheimer's disease, respectively (Liz et al., 2010).

A neuroprotective role of TTR has also been described under conditions of cerebral ischemia in mice deficient for heat shock transcription factor 1 (HSF1), an activator of heat-shock proteins. Under conditions of compromised heatshock response, TTR from CSF contributes to control neuronal cell death, edema and inflammation, thereby influencing the survival of endangered neurons in cerebral ischemia (Santos et al., 2010). More recent work, indicates that TTR acts as neurotrophic factor, through interaction with megalin, stimulating neurite outgrowth and promoting neuroprotection in ischemic conditions (Gomes et al., 2016, 2019). 


\section{TRANSTHYRETIN-RELATED AMYLOIDOSIS}

Transthyretin amyloidosis (ATTR amyloidosis) is a group of diseases in which TTR variants (ATTRv) or even the wildtype protein (ATTRwt) aggregate and form amyloid fibrils that deposit extracellularly in tissues (Sipe et al., 2016). These are, respectively, hereditary and non-hereditary forms of the disease. The non-hereditary form is related to alterations of environmental conditions and aging leading to the aggregation and fibril formation of wild type TTR, ATTRwt (Sipe et al., 2016). Thus, ATTRwt amyloidosis is mainly an age-related disorder, affecting $12-25 \%$ of the population over 80 yearsold and is characterized by ATTRwt deposition, particularly in the heart, affecting cardiac functions (Westermark et al., 2003). In contrast, the hereditary forms of the disease, ATTRv amyloidosis result from single point mutations in the coding region of the TTR gene, mainly producing less stable variant proteins with an altered amino acid in the polypeptide chain, ATTRv (Saraiva, 1995). Accordingly, to date, more than 140 mutations on the TTR gene have been described (http:// amyloidosismutations.com/mut-attr.php) (Connors et al., 2003). Among these, only about 15 TTR variants are reported as nonamyloidogenic, while most TTR point mutations induce systemic amyloidosis with predominant neuropathic (Plante-Bordeneuve and Said, 2011), or cardiac phenotypes (Rapezzi et al., 2010). However, most of the variants have been associated with a mixed phenotype, characterized by varying degrees of neurological and cardiac involvement (Conceicao et al., 2019). Less frequently, manifestations of ATTR amyloidosis include vitreous opacities (Ando et al., 1997) and, in rare cases, leptomeningeal amyloidosis (Maia et al., 2015).

The substitution of valine for methionine at position 30 (V30M) in the TTR polypeptide chain was the first mutation to be identified and, is the most common mutation associated with ATTR polyneuropathy (ATTR-PN) (previously designated familial amyloid polyneuropathy-FAP) (Saraiva et al., 1984). This life-threatening disease, first described by Corino de Andrade (Andrade, 1952) mainly affects both peripheral and autonomic nervous system, being sensorimotor polyneuropathy, autonomic dysfunction and gastrointestinal tract disturbances the major clinical manifestations which may lead to death within 10 years after disease onset if not treated (Ando et al., 2005; Conceicao et al., 2016).

The prevalence of ATTR V30M amyloidosis is estimated to be $0.87-1.1$ per 1000000 individuals (Adams et al., 2014) and the disease has been considered endemic in the north of Portugal (Sousa et al., 1995), Japan (Kato-Motozaki et al., 2008), and Sweden (Sousa et al., 1993). Individuals from Portugal and some provinces in Japan typically manifest early-onset and high-penetrance phenotype, whereas people in Sweden and, also in other Japanese regions usually present late-onset and lowpenetrance disease (Plante-Bordeneuve and Said, 2011).

Besides peripheral neuropathy, cardiomyopathy is also one of the major clinical manifestations of ATTR amyloidosis (ATTRCM) (Suhr et al., 2003). In addition to ATTRwt, which is the main cause of ATTR-CM, as mentioned above, some non-V30M mutations on TTR gene also lead to the development of cardiac symptoms (Westermark et al., 1990). In particular, TTR V122I is the most common variant responsible for ATTR-CM being almost exclusively found in 3-4\% of African-Americans and, the predominant phenotype associated with this mutation is severe restrictive cardiomyopathy with late-onset, i.e., occurs mainly after the age of 60 , without neurological symptoms (Jacobson et al., 1997; Quarta et al., 2015; Buxbaum and Ruberg, 2017). There are also other TTR variants responsible for the development of cardiac amyloidosis, such as T49A, S50I, T60A, I68L, and L111M (Rapezzi et al., 2015; Sekijima, 2015).

In patients with ATTR-CM, amyloid fibrils can infiltrate any or all cardiovascular structures including conduction system, the atrial and ventricular myocardium, valvular tissue and, the coronary and large arteries (Falk and Dubrey, 2010). Myocardial infiltration results in progressive increase in the thickness of left and right ventricular walls and of the interatrial septum, ultimately leading to heart failure (Rapezzi et al., 2010).

The diagnosis of ATTR-CM firstly includes echocardiogram and electrocardiogram (Donnelly and Hanna, 2017). However, myocardial scintigraphy using bone avid tracers, in particular, technetium-based isotypes, such as $99^{\mathrm{m}}$ technetium $3,3-$ diphosphono-1,2-propanodicarboxylic acid, pyrophosphate and hydroxymethylene diphosphonate revealed high sensitivity and specificity to cardiac ATTR amyloid deposits. In fact, these agents allow to identify deposits before increasing myocardial wall thickness, contributing to early diagnosis of ATTR-CM (Maurer et al., 2019). In addition, alterations in the values of cardiac biomarkers have also been increasingly helpful on the management of ATTRCM. Indeed, clinical data from Patel and Hawkins indicate that substantial ATTR amyloid deposits accumulating in the cardiac tissue are accompanied by a moderate increase in serum levels of NT-proBNP concentration (Patel and Hawkins, 2015).

Approximately, one-fourth of the amyloidogenic TTR mutations originates vitreous amyloid, namely F33I, R34G, L35T, I84S, and T114C (Sekijima, 2015). It has been postulated that vitreous amyloid is the result of local TTR synthesis in the RPE cells in the eye (Ando et al., 1997). Similarly to vitreous, also leptomeningeal amyloidosis may be related to local TTR synthesis, in this case by choroid plexus and, amyloid deposition mainly occurs in the media and adventitia of medium-sized and small arteries, arterioles and veins of the cortex and leptomeninges. These amyloid infiltrations induce cerebral infarction, cerebral hemorrhage, subarachnoid hemorrhage and hydrocephalus, ultimately leading to serious central nervous dysfunctions, namely ataxia and dementia (Maia et al., 2015; Sekijima, 2015). Till now, leptomeningeal amyloidosis is mainly associated with D18G, A25T, and T114C TTR mutations (Sekijima, 2015). However, in some cases, leptomeningeal amyloidosis may also develop in patients with V30M mutation (Maia et al., 2015). 


\section{TTR AMYLOID FORMATION}

The hallmark of ATTR amyloidosis is the extracellular deposition of aggregated TTR or TTR fibrils in tissues. The process of TTR aggregation and fibril formation is not completely elucidated however biochemical and biophysical evidences indicate that the tetrameric form of TTR becomes unstable and the protein dissociates into dimers and monomers presenting a partially unfolded conformation which self-assemble into toxic nonfibrillar aggregates and, later into amyloid fibrils that accumulate as amyloid deposits throughout the body (Quintas et al., 2001; Cardoso et al., 2002).

In vivo the amyloid deposits are composed also by other proteins such as serum amyloid $\mathrm{P}$ component (SAP) and proteoglycans (Benson et al., 2018a). In the case of ATTR amyloidosis, TTR in the amyloid deposits might be in its intact form meaning as full-length protein and as TTR fragments suggesting that proteolysis might contribute as a mechanism of amyloid formation.

Knowledge of the mechanisms involved in TTR amyloid formation allows establishing therapeutic targets to avoid and/or halt the progression of disease. In this sense several therapeutic strategies have been pursued targeting different stages of the process of amyloid formation or clearance of pre-formed fibrils. The main targets have been lowering or silencing TTR, TTR stabilization, inhibition of TTR fibril formation and fibril disruption that will be discussed below.

\section{ATTR AMYLOIDOSIS THERAPIES TARGETING TTR SYNTHESIS}

TTR variants are the main component of amyloid deposits in ATTRv amyloidosis, therefore abolishment of TTR synthesis was one of the first proposed therapeutic approaches in these diseases. Since the liver is the main organ producing and secreting TTR into blood, liver transplant emerged as a possible therapeutic strategy for ATTR amyloidosis (Lewis et al., 1994). Indeed, orthotopic liver transplant (OLT) was shown to arrest disease progression through suppression of mutant TTR from circulation and has been the most effective treatment for ATTR amyloidosis (Benson, 2013; Ericzon et al., 2015). Despite the favorable prognosis observed in transplanted patients, there are still some concerns and long-term complications since the previously existing hereditary amyloid deposits may recruit newly circulating TTR wt promoting amyloid growth and, ultimately resulting in disease progression (Maurer et al., 2016; Saelices et al., 2018). Additionally, there are reports of continuous amyloid deposition even after liver transplantation, mainly in cardiac tissue of transplanted TTR V30M carriers (Okamoto et al., 2011), as well as in the vitreous humor (Munar-Ques et al., 2000; Ando et al., 2001) and leptomeninges (Sekijima et al., 2016). This may be due to the recruitment of newly circulating TTR wt by the previously existing amyloid deposits, mainly in the heart or due to the local TTR synthesis in the eye and choroid plexus of the brain, in either case this might result in amyloid growth and disease progression (Maurer et al., 2016; Saelices et al., 2018). On the other hand, in the case of domino liver transplant (DLT), in which the liver excised from ATTRv patient is transplanted to patients with severe liver disease, the recipients developed symptoms related to ATTR amyloidosis (Stangou et al., 2005; Goto et al., 2006; Barreiros et al., 2010). In addition, post-mortem analysis indicated that systemic amyloid deposition occurred before the appearance of the symptoms (Koike et al., 2011). Interestingly, the clinical manifestations of acquired ATTR amyloidosis after DLT are predominantly related to sensory deficits contrary to the predominant autonomic symptoms in the donors (Stangou et al., 2005; Goto et al., 2006; Barreiros et al., 2010).

The previous findings of continuous amyloid deposition, even after OLT, as well as the knowledge that TTR wt may also aggregate into amyloid fibrils (Westermark et al., 2003), led to an increasing interest in less invasive treatments aiming also to arrest TTR synthesis through gene silencing as a new strategy for the treatment of ATTR amyloidosis. Two different genesilencing approaches have been developed. One is based on antisense nucleotides (ASOs) and the other on small-interfering RNA (siRNA) (Gertz et al., 2019).

TTR specific siRNAs were firstly tested in mouse models of ATTR amyloidosis and a reduction on amyloid deposition was observed, inducing ATTR amyloid regression (Butler et al., 2016). Different siRNAs with similar mechanism of action were further assessed, in particular patisiran and revusiran, being patisiran the one selected for phase III clinical trials (Coelho et al., 2013). Prolonged administration of patisiran in an openlabel study for an extended period of 29 months demonstrated a consistent lowering of plasma TTR levels resulting in disease stabilization and absence of major safety concerns, confirming its indication for ATTR amyloidosis therapy (Adams et al., 2018).

More recently, a novel liver-directed siRNA conjugate, vutrisiran, has been formulated. Vutrisiran is a GalNAcsiRNA conjugate presenting improved pharmacokinetic and pharmacodynamic properties allowing potent and sustained TTR reduction and an acceptable safety profile with mild treatmentrelated adverse effects as found in a phase I clinical trial enrolling healthy individuals (Habtemariam et al., 2020). These improved characteristics suggest vutrisiran as a novel promising therapy for the treatment of ATTR amyloidosis.

Furthermore, a second-generation ASOs (e.g., IONIS-TTRRx or Inotersen) was reported to be effective by decreasing TTR plasma levels in both monkeys and ATTR I84S transgenic mice. Experiments in healthy humans also revealed a decrease in TTR wt plasma concentrations in a dose-dependent manner (Ackermann et al., 2016). More recent results from phase 3 clinical trial studies with these two gene silencers, patisiran and inotersen, reveal that both are able to efficiently reduce TTR synthesis and arrest disease progression though with some differences in the form and frequency of the therapeutic administration and safety monitoring (Adams et al., 2018; Benson et al., 2018b; Gertz et al., 2019; Koike and Katsuno, 2020). 


\section{ATTR AMYLOIDOSIS THERAPIES TARGETING AMYLOID FORMATION}

Several compounds have been suggested for the treatment of ATTR amyloidosis by targeting different steps of the amyloid formation. The main steps include TTR stabilization, inhibition of oligomerization and fibril disruption. The most relevant compounds are listed in Table $\mathbf{1}$ and will be discussed in the following sections. In the recent years, computational studies, such as molecular dynamics (MD) simulations, molecular docking and quantitative structural-activity relationships (SAR) have been used as complement of experimental approaches to better understand TTR monomer misfolding mechanismsdriving TTR amyloidogenesis (Zhou et al., 2019), as well as the binding of small molecules to TTR (Dessi et al., 2020). Indeed, these in silico experiments have been essential to obtain a more detailed information about structural changes in biomolecules and, have been used to determine the structural dynamics of TTR (Ortore and Martinelli, 2012; Zhao and Lei, 2014), which in turn will be particularly relevant to the development of more targeted and effective therapies for the treatment of ATTR amyloidosis.

\section{TTR Stabilization}

TTR tetramer stability is a determinant factor conditioning tetramer disassembly, the rate-limiting step for aggregation and amyloid fibrils formation (McCutchen et al., 1993; Quintas et al., 1999). Accordingly, the development of small molecules able to stabilize the TTR tetramer, preventing its dissociation into monomers, has been recognized as a great therapeutic strategy for the treatment of ATTR amyloidosis. The design of these molecules was based on the affinity of $\mathrm{T}_{4}$ to bind to the central pocket of the TTR tetramer inhibiting its dissociation (Miroy et al., 1996). Based on the capacity of the nonsteroidal anti-inflammatory drugs (NSAIDs), to bind to the $\mathrm{T}_{4}$-binding channel in TTR (Baures et al., 1999; Miller et al., 2004), the first drug to be tested was diflunisal, which was reported as an effective stabilizer of the TTR tetramer in plasmas from ATTR-PN patients (Tojo et al., 2006). Then, ATTRv patients were randomly assigned to receive diflunisal for 2 years and, in fact, the use of diflunisal reduced the rate of progression in neurologic impairment and preserved the quality of life of patients comparatively to placebo group (Berk et al., 2013) and ameliorated the autonomic symptoms in ATTRv patients (Takahashi et al., 2014). However, diflunisal administration to these patients induced long-term side effects, namely impaired renal function and thrombocytopenia (Sekijima et al., 2015), which may compromise its clinical value.

Following, other pharmacologic molecules, such as tafamidis (a benzoaxazole derivative) (Vyndaqel ${ }^{\circledR}$ ) have been proposed through a structure-based drug design approach to select compounds to occupy these $\mathrm{T}_{4}$-binding sites, kinetically stabilizing the TTR tetramer, and ultimately resulting in a decrease in the rate of amyloid fibril formation in vitro (Bulawa et al., 2012). One of the major concerns about the use of tafamidis in ATTRv patients is related to potential metabolic side effects, since it could interfere with $\mathrm{T}_{4}$ delivery throughout the body. However, clinical trials have found minimal evidences about this concern because thyroxine binding globulin (TBG), rather than TTR, transports the majority of the circulating $\mathrm{T}_{4}$ ( 75\%) (Refetoff, 2000; Coelho et al., 2012). Tafamidis has gained approval for the treatment of ATTRv amyloidosis in several countries, including in the European Union, Mexico, Argentina, Japan and more recently also in the USA for the treatment of ATTR-CM (Coelho et al., 2016). Moreover, an open-label extension study for 6 years also revealed the slowing of neuropathy progression without unexpected adverse effects (Barroso et al., 2017). Recently, the effects of tafamidis on ATTRCM were also evaluated in both ATTRv and ATTRwt patients in a phase III clinical trial significantly reducing mortality and cardiovascular-related hospitalizations (Maurer et al., 2018).

Most studies for the development of efficient TTR stabilizers were based on rational ligand design and, thus most of the stabilizers are, in general, halogenated biaryl analogs of $\mathrm{T}_{4}$, many resembling NSAIDs. However, these molecules act as cyclooxygenase (COX) inhibitors increasing the risk of severe cardiovascular events therefore being contraindicated in patients with ATTR-CM (Mukherjee et al., 2001). Moreover, highthroughput screening studies pointed out a new compound, AG10, as an effective and selective stabilizer of the cardiac TTRwt and TTR V122I protecting human cardiomyocytes from TTR amyloid toxicity (Alhamadsheh et al., 2011; Penchala et al., 2013). Interestingly, structural studies revealed that AG10 is unique in its capacity to form hydrogen bonds with the same serine residues at position 117 that stabilize the non-amyloidogenic TTR T119M variant (Miller et al., 2018). Recent results from phase II clinical trials revealed that AG10 has the potential to be safe and effective for the treatment of ATTR-CM patients either carrying mutant or TTR wt. Phase III clinical trials with AG10 are ongoing (Judge et al., 2019).

In addition, based in its molecular structure, tolcapone, an FDA-approved drug for the treatment of Parkinson's disease, has been repurposed for the treatment of ATTR amyloidosis. Tolcapone specifically binds to TTR in human plasma and, stabilizes the native TTR tetramer in vivo in mice and humans. Furthermore, it was also demonstrated that the binding of tolcapone to the recombinants TTR wt and TTR V122I, at the $\mathrm{T}_{4}$-binding channel is stronger comparatively to tafamidis (Sant'Anna et al., 2016). These results pointed-out tolcapone as a strong candidate for the treatment of ATTR polyneuropathy and, in fact, it has gained clinical interest and it already passed phase I/II clinical trials (Gamez et al., 2019). A very recent work on the structural characterization of Tolcapone-TTR complexes demonstrates high stabilization and binding affinity of Tolcapone to TTR variants associated with leptomeningeal amyloidosis. These characteristics in association with its ability to cross the blood-brain barrier suggests its particular indication for therapeutic intervention in this type of amyloidosis (Pinheiro et al., 2020).

Contrarily to the above-mentioned compounds, palindromic ligands, such as mds84, rapidly bind simultaneously to both $\mathrm{T}_{4^{-}}$ binding sites in each tetrameric TTR molecule, which would overcome the problems of negative cooperativity of the binding of the existing drugs, such as tafamidis. Mds84 binds to the native TTR wt in whole serum and, more effectively to 
the amyloidogenic TTR variants, promoting the stabilization of the TTR tetramer (Kolstoe et al., 2010; Corazza et al., 2019).
Some plant polyphenols which may be part of our diet have also been reported as TTR tetrameric stabilizers. In particular, epigallocatechin-3-gallate (EGCG) and curcumin, the major

TABLE 1 | Compounds proposed for the treatment of ATTR amyloidosis.

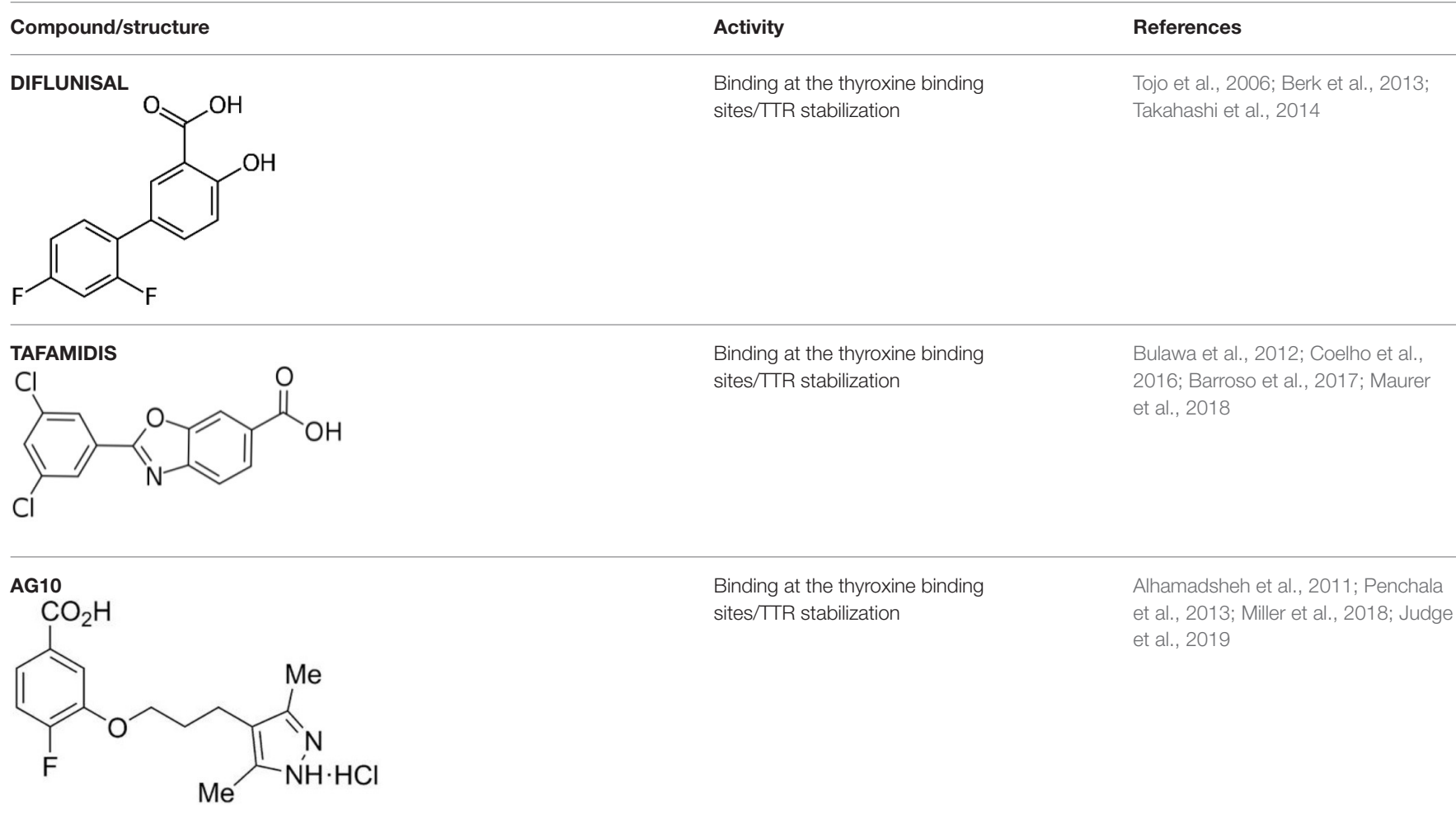<smiles>Cc1ccc(C(=O)c2cc(O)c(O)c([N+](=O)[O-])c2)cc1</smiles>

Binding at the thyroxine binding sites/TTR stabilization
Sant'Anna et al., 2016; Gamez et al., 2019; Pinheiro et al., 2020<smiles>CCCCCCCCCCCCOc1c(Cl)cc(Nc2ccccc2C(=O)O)cc1Cl</smiles>

Binding at the thyroxine binding sites-bivalent ligand/TTR stabilization
Kolstoe et al., 2010; Corazza et al., 2019

\section{CURCUMIN}<smiles>COc1cc(/C=C/C(C)=O)ccc1O</smiles>

Binding at the thyroxine binding sites/TTR stabilization
Ferreira et al., 2011, 2013, 2016, 2019 


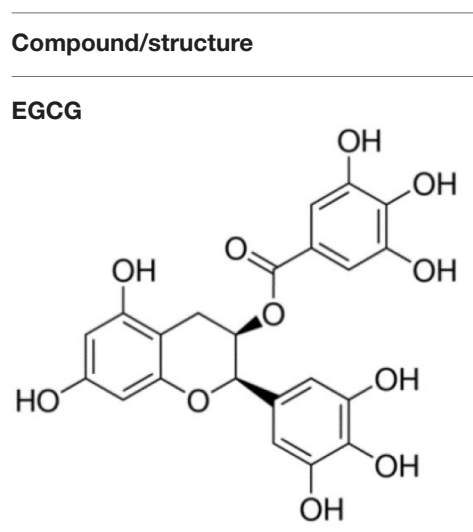

Molecular tweezer CLR01

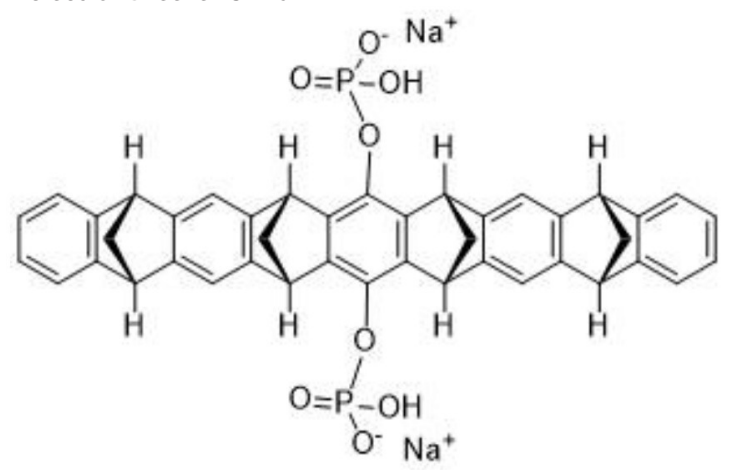

Inhibition of aggregation (oligomerization)/Disruption of aggregates
TTR stabilization /inhibition of aggregation (oligomerization)/Disruption of aggregates
References

Ferreira et al., 2009, 2011, 2012<smiles>[2H]OC(=O)N(C)[C@@H]1C(O)=C(C(N)=O)C(=O)[C@@]2(O)C(O)=C3C(=O)c4c(O)cccc4[C@H](C)[C@]3(C)[C@@H](O)[C@@]12C</smiles>

Disruption of TTR aggregates

Cardoso et al., 2003, 2008; Cardoso and Saraiva, 2006

components of green tea and turmeric, respectively, were able to effectively stabilize the TTR tetramer in human plasmas from both V30M carriers and controls (Ferreira et al., 2011), as well as in plasmas from transgenic mice carrying human TTR V30M variant (HM30 mice) (Ferreira et al., 2012, 2013). It should be noted that these compounds exhibit different ways of action. Curcumin competes with $\mathrm{T}_{4}$ for the binding to TTR, meaning that it binds at the $\mathrm{T}_{4}$ binding sites, whereas EGCG stabilizes TTR through binding at the surface of the TTR molecule in particular at two binding sites at the dimer-dimer interface exerting an effect similar to a cross-linker. Low bioavailability and low specificity of binding seem to be relevant conditioning factors of their effects in vivo in humans (Kristen et al., 2012; aus dem Siepen et al., 2015; Cappelli et al., 2018).

\section{Inhibition of TTR Aggregation Into Amyloid Fibril}

TTR stabilizers, as the above-mentioned small molecule compounds, including EGCG, curcumin, tolcapone and mds84 have also been reported as inhibitors of TTR amyloid formation as consequence of their effect on the first step of TTR aggregation.

Ferreira et al. firstly described EGCG as a strong inhibitor of TTR aggregation in vitro (Ferreira et al., 2009), by maintaining most of the protein in a non-aggregated soluble form. EGCG also suppressed the amyloid fibril formation pathway in a cell culture system (Ferreira et al., 2009). Later, the role of EGCG on the inhibition of amyloid fibril formation in vivo using a wellcharacterized transgenic murine model of ATTR-PN was also demonstrated. EGCG reduced, in about 50\%, the deposition of TTR toxic aggregates in the gastro-intestinal tract and peripheral nervous system (PNS), with a concomitant decrease in the expression of both non-fibrillar-related biomarkers and amyloid deposition markers (Ferreira et al., 2012).

Similar studies using curcumin demonstrated suppression of fibril formation in vitro through the generation of small "offpathway" oligomers (Ferreira et al., 2011) and inhibited this process in transgenic mice carrying human TTR V30M variant. In fact, immunohistochemical analysis of mice tissues revealed 
that dietary curcumin decreased TTR load in as much as $70 \%$ and lowered the cytotoxicity associated with TTR aggregation (Ferreira et al., 2013). Later, it has been shown that dietary curcumin decreases TTR deposition and associated toxicity in the dorsal root ganglia and stomach of aged mice carrying human TTR V30M variant (Ferreira et al., 2016).

Furthermore, synthetic compounds such as tolcapone and mds84 effectively inhibited the process of TTR fibril formation in vitro (Kolstoe et al., 2010; Sant'Anna et al., 2016) and, tolcapone was also able to suppress TTR toxicity in cellular models (Sant'Anna et al., 2016).

However, some inhibitors of amyloid formation might act on a different step of the cascade leading to fibril formation that includes, for instance, the polymerization of the intermediate species originating aggregates that evolve to amyloid fibrils. That is the case of the molecular tweezer CLR01 (Sinha et al., 2011). This is a synthetic compound that through binding to positively charged amino acids, in particular lysine and arginine residues in the terminal beta-strands of TTR, inhibit the tight alignment of protofilaments characteristic of amyloid formation. Thus, the molecular tweezer CLR01 inhibited TTR aggregation in vitro and also in vivo as demonstrated in a study in which TTR V30M mice treated with CLR01 presented decrease of TTR deposition and of associated biomarkers (Ferreira et al., 2014). However, this compound presents limitations related to the low binding affinity to proteins and to its formulation needing improvement of pharmacologic properties.

\section{Disruption of Aggregates}

The role of anthracyclines and, in particular of $4^{\prime}$-iodo- $4^{\prime}$ deoxydoxorubicin on the reabsorption of amyloid deposits was related to the almost planar structure of these compounds and the cross $\beta$-pleated structure characteristic of all amyloid fibrils (Merlini et al., 1995). Furthermore, doxycycline, a member of tetracycline antibiotics family, structurally homologous to the anthracyclines, was found to be particularly effective on the disruption of TTR amyloid fibrils in vitro (Cardoso et al., 2003). In addition, in vivo studies on transgenic mice carrying human TTR V30M variant supported the previous in vitro findings. Doxycycline was administered to old transgenic mice and, tissue analysis revealed Congo red positive staining only for the nontreated animals from the control group. Additionally, a decrease in several markers associated with TTR amyloid deposition was also reported (Cardoso and Saraiva, 2006; Cardoso et al., 2008). The recent development of doxycycline conjugates, namely polyglutamate-doxycycline, demonstrated an enhanced effect in the clearance of fibrils comparatively to non-conjugated doxycycline only (Conejos-Sanchez et al., 2015).

Since doxycycline has effect only in advanced phases of the amyloidogenic cascade it has been proposed that it could be combined with another drug targeting an earlier phase of the amyloid fibrils assembly (Cardoso et al., 2010). In this sense, tauroursodeoxycholic acid (TUDCA), a hydrophilic biliary acid derivative, gained particular clinical interest for the treatment of ATTR amyloidosis since it has been previously referred to cause a decrease in the deposition of toxic pre-fibrillar TTR oligomers and to reduce the expression of several apoptotic and oxidative biomarkers associated with ATTR amyloid deposition in transgenic murine models treated with TUDCA (Macedo et al., 2008; Cardoso et al., 2010). Clinical trials of combined doxycycline and TUDCA are underway and preliminary results indicate positive effects though more results are necessary to evaluate the impact of this therapeutic approach in disease progression (Obici and Merlini, 2014).

Moreover, some therapeutic compounds are classified as multi-target disease agents, performing a role in different steps of amyloid fibril formation. For instance, compounds such as EGCG and curcumin besides its effects as inhibitors of aggregation act also as disruptors of TTR amyloid deposits. In fact, both natural polyphenols, EGCG and curcumin, efficiently disaggregated preformed TTR amyloid fibrils (Ferreira et al., 2011) (Ferreira et al., 2019). Recent studies using transgenic murine models pointed out both curcumin and TUDCA as modulators of cellular autophagy processes, which are involved in the clearance of large protein aggregates (Teixeira et al., 2016).

\section{Immunotherapy}

Immunotherapy is another therapeutic strategy for the treatment of ATTR amyloidosis, which still remains under investigation. Specific antibodies targeting TTR monomers, oligomers or amyloid aggregates may prevent TTR fibrillogenesis. As a first approach, a structure-based strategy was used to develop a TTR conformation-specific antibody targeting pre-fibrillar, misfolded TTR intermediates without recognizing native tetrameric TTR. This is achieved since the antibody (misTTR) targets the residues 89-97 in the polypeptide chain, which are buried in the TTR tetramer, but it is exposed in the monomer, inhibiting fibrillogenesis of misfolded TTR under micromolar concentrations (Galant et al., 2016). This antibody has already entered into phase I clinical trials in ATTRv patients (Macedo et al., 2020).

\section{CONTRIBUTION OF TTR PROTEOLYSIS TO AMYLOID FORMATION}

Since a long time ago, TTR proteolysis has been suggested to be involved in the mechanisms driving TTR-related amyloidosis (Pitkanen et al., 1984). Therefore, by understanding in detail the molecular mechanisms implicated in the pathophysiology of ATTR amyloidosis, it would be possible to develop new targeted therapies to improve the patients' outcomes.

Several evidences suggest the existence of different types of TTR amyloid fibrils in a range of tissues. In fact, amyloid deposits might be composed by a mixture of both cleaved and full-length TTR (type A) or full-length TTR only (type B). The resulting amyloid deposits are different. Type A fibrils are shorter and exhibit weaker affinity for Congo Red staining than type B fibrils, which are longer, slender and strongly stain with Congo Red (Bergstrom et al., 2005; Ihse et al., 2008, 2011).

Different amyloidogenic fragments may be found in different tissues and could be associated either with ATTRwt or ATTRv amyloidosis (Suhr et al., 2017). Vitreous TTR appeared to be fragmented between the residues Lys48-Thr49, whereas cardiac 
TTR may be cleaved at multiple sites between the 46-52 amino acid residues in polypeptide chain (Liepnieks et al., 2006). However, peptide 49-127 C-terminal fragment is the main component of ex vivo TTR amyloid fibrils in tissue biopsies of cardiac deposits, which is further associated with poor clinical prognosis, often with rapidly progressive cardiac involvement, even after liver transplantation (Gustafsson et al., 2012; Ihse et al., 2013).

The protease responsible for TTR cleavage has not yet been identified. However, the highly specific fragmentation pattern suggests that it could be a trypsin-like serine protease. The three-dimensional structure of this protein region is solvent exposed and potentially accessible for cleavage. In accordance, all amyloidogenic TTR variants showed an increased main chain solvent exposure comparatively to both native and non-amyloidogenic variants, which may result in increased susceptibility to proteolysis (Schormann et al., 1998).

Recent in vitro studies, using recombinant trypsin, revealed that the proteolysis/fibrillogenesis pathway is common to several amyloidogenic TTR variants and, the process of cleavage and release of the 49-127 TTR fragment is faster for the highly amyloidogenic variant, TTR S52P, than for the other TTR variants analyzed (Mangione et al., 2014; Marcoux et al., 2015). It requires the action of biomechanical forces provided by sheer stress of physiological fluid flow and, importantly, the non-amyloidogenic TTR T119M is neither cleaved nor generates amyloid fibrils under these conditions. These studies also demonstrated that the TTR stabilizers, mds84, tolcapone, diflunisal and tafamidis, inhibited TTR proteolysis resulting in the inhibition of aggregation. However, the maximum inhibition is only achieved when both $\mathrm{T}_{4}$-binding sites in central hydrophobic channel are simultaneously occupied by small ligands (Mangione et al., 2014; Verona et al., 2017). In opposition, natural TTR ligands, $\mathrm{T}_{4}$ and RBP, were not able to inhibit TTR cleavage. Nevertheless, binding of RBP, but not $\mathrm{T}_{4}$, effectively inhibited the subsequent formation of amyloid fibrils (Mangione et al., 2014).

Due to the exclusive duodenal location of trypsin, it is unlikely that it may contribute to the development of systemic TTR amyloidosis in vivo. In silico studies recently pointed out plasmin as a plausible pathophysiological candidate protease involved in the process of TTR amyloid formation (Mangione et al., 2018). Furthermore, the ubiquitous distribution of plasmin, its structural similarities to trypsin (Mangione et al., 2018) and the reported activation of plasminogen activation system (PAS) in other amyloid-related disorders, such as Alzheimer's disease (Tucker et al., 2000) and immunoglobulin light chain (AL) amyloidosis (Mumford et al., 2000; Bouma et al., 2007; Uchiba et al., 2009) also indicate that this protease could perform a key role in TTR amyloidogenesis.

Recent studies showed that amorphous protein aggregates are degraded by plasmin, releasing smaller soluble protein fragments, which are cytotoxic in vitro for both endothelial and microglial cells (Constantinescu et al., 2017).

Plasmin, similarly to trypsin, selectively cleaves TTR S52P variant, at Lys48-Thr49 peptide bond under physiological conditions in vitro being, both the TTR fragments and full-length protomers readily released from the homotetramer and incorporated into amyloid fibrils, morphologically identical to ex vivo TTR amyloid (Mangione et al., 2018). Concerning these observations, a hypothetical model for the role of plasmin-mediated proteolysis on TTR fibrillogenesis has been proposed. In this model, circulating TTR can diffuse toward the extracellular compartment, be entrapped in the fibrin clot or escape from it. Upon plasminogen activation, TTR may be cleaved and then dissociate into a mixture of both truncated and full-length TTR, which ultimately assemble into amyloid fibrils and deposit at the extracellular space (Mangione et al., 2018).

Altogether these evidences seem to point out the importance of lysine (Lys) residues for the pathogenicity of ATTR amyloidosis as it has been described for other amyloid disorders (Sinha et al., 2011). By targeting the Lys residues using synthetic Lys specific molecular tweezers (e.g., CLR01), the process of TTR proteolysis could be effectively inhibited through its binding to Lys48, which seem to be target of the protease responsible for TTR cleavage. This could be particularly important for the treatment of both ATTR-CM and vitreous amyloidosis, since the 49-127 TTR fragment has been frequently encountered in the amyloid deposits in both cases.

Despite the increasing interest on TTR proteolysis as leading mechanism-driving ATTR amyloidosis, some questions remain to be answered. Though, it is still unknown whether TTR fragmentation occurs prior or after aggregation and, where it occurs, in circulation or at the site of deposition, an increase of the proteolytic activity in plasmas from ATTR patients comparatively to healthy controls, suggesting that the process occurs in the bloodstream before fibril formation (da Costa et al., 2015).

\section{EXTRACELLULAR CHAPERONES AS REGULATORS OF ATTR AMYLOIDOSIS}

The disruption of the protein folding quality control mechanisms is also an underlying cause of ATTR amyloidosis. Recently, some studies revealed the existence of a growing family of extracellular chaperones in body fluids, which selectively bind to exposed hydrophobic residues in misfolded proteins in order to prevent their toxicity upon aggregation into insoluble deposits (Wyatt et al., 2013).

Among those extracellular chaperones, haptoglobin, alpha-2microglobulin (A2M) and clusterin were found to be increased in plasmas from ATTR patients (da Costa et al., 2015). While haptoglobin and A2M, were previously described as effective in the inhibition of stress-induced aggregation of a number of unrelated target proteins (Yerbury et al., 2005; French et al., 2008), clusterin is an ubiquitous highly conserved secreted protein (Wyatt et al., 2009), which inhibits protein aggregation in an ATP-independent manner upon its binding to misfolded proteins, such as $\alpha$-synuclein and $\beta$-amyloid peptide, producing soluble, high molecular complexes (Matsubara et al., 1996; Poon et al., 2000; Yerbury et al., 2007).

The role of clusterin on the clearance of extracellular aggregates has also been investigated in ATTR-PN (Lee et al., 
2009; Magalhaes and Saraiva, 2011). In vitro studies using neuroblastoma cells incubated with TTR oligomers revealed intracellular clusterin overexpression and increased levels of clusterin secreted to the culture medium. An overexpression of clusterin in tissues with TTR deposition was found in mice carrying human TTR V30M in HSF-1 null background, which exhibit early and extensive non-fibrillar TTR deposition in the gastrointestinal tract and in the peripheral and autonomic nervous system. In addition, in human nerve, clusterin colocalizes either with fibrillar or non-fibrillar TTR deposits as detected by double immunostaining (Magalhaes and Saraiva, 2011).

Clusterin was also found in cardiac TTR amyloid deposits from patients with ATTRwt and ATTRv (Greene et al., 2011) and, later, experiments using circular dichroism spectroscopy revealed that clusterin preferentially stabilizes monomeric TTR leading to the appearance of increasingly stable conformations under acid stress. Additionaly, clusterin interacts also with high molecular weight TTR aggregated species and, these interactions with both monomeric and oligomeric TTR proceed in a cooperative manner in the presence of the TTR tetramer stabilizer, diflunisal. Altogether these observations suggest a novel synergistic treatment for ATTR amyloidosis using both diflunisal and clusterin for the removal of misfolded and aggregated TTR (Greene et al., 2015). Accordingly, preliminary data revealed a temporal increase in serum clusterin levels in patients treated with diflunisal at 1-year follow-up compared to baseline. In opposition, patients who were not treated with diflunisal demonstrated decreased clusterin levels at annual evaluation. Interestingly, a positive correlation between clusterin and TTR levels was found at baseline suggesting that soluble tetrameric TTR decreases as more of the native protein dissociates and forms species, overwhelming the protein folding capacity of clusterin leading to a reduction in circulating levels of this molecular chaperone and, the treatment of the ATTR patients with diflunisal lead to a partial recovery of serum clusterin levels (Torres-Arancivia and Connors, 2019). These results are in accordance with previous studies reporting the beneficial effects of diflunisal for the treatment of ATTRv amyloidosis.

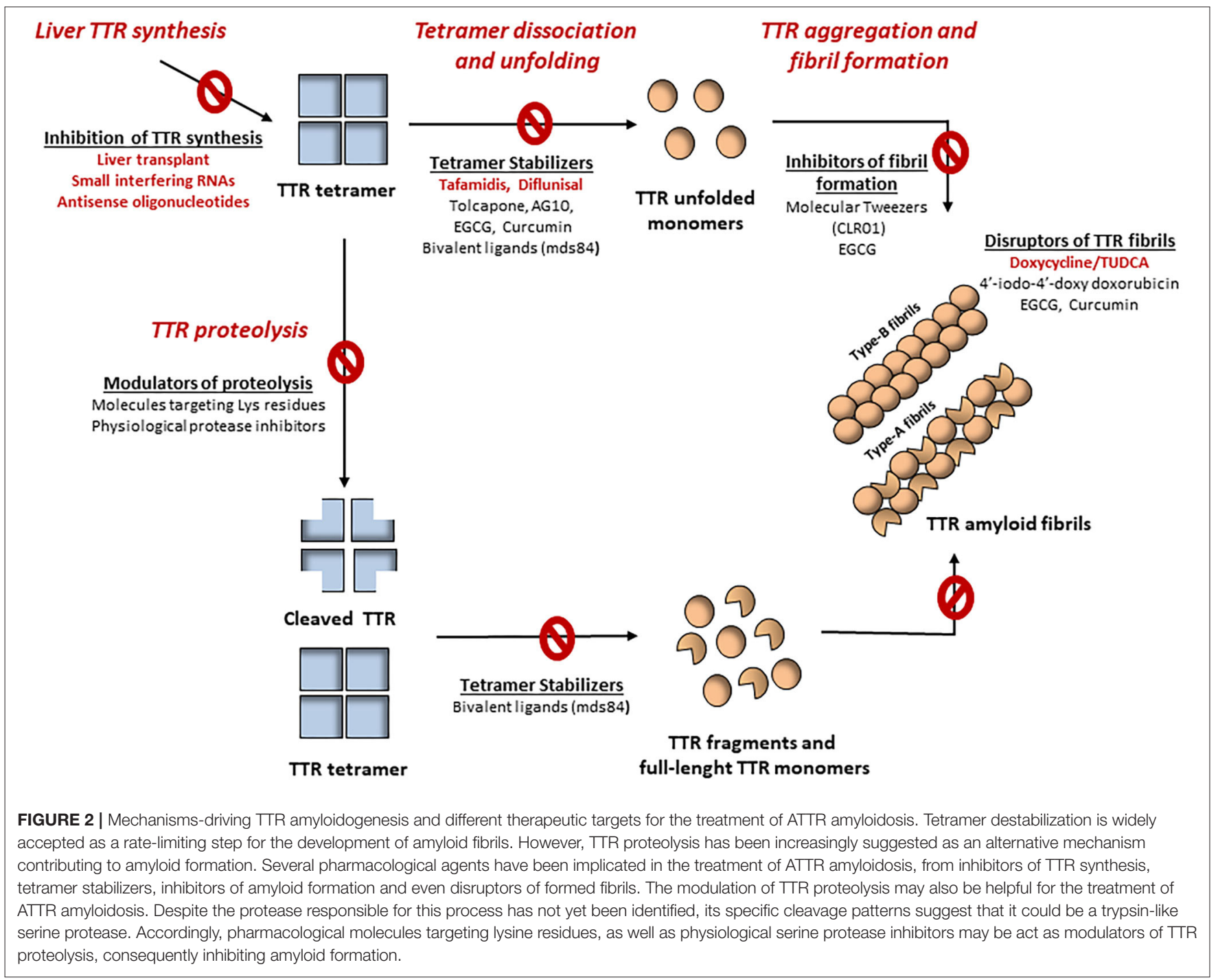




\section{CONCLUSION AND FUTURE PERSPECTIVES}

ATTR amyloidosis is an under-recognized disease which is characterized by extracellular deposition of TTR aggregates in several organs, being polyneuropathy and cardiomyopathy the major clinical manifestations. The mechanism by which the tetramer disassembles and aggregates into amyloid fibrils has been considered the main driver of the disease. However, TTR proteolysis, namely occurring in the cardiac tissue, as well as its modulation have been increasingly documented as fundamental for understanding the development and progression of ATTR amyloidosis.

Many therapeutic approaches have been suggested for the treatment of ATTR amyloidosis targeting different steps of the pathology. Those therapies include interventions from the synthesis of the TTR variants through liver transplant or gene silencing therapies, to TTR stabilization, inhibition of aggregation, disruption of amyloid fibrils and clearance of amyloid deposits. The main targets for intervention on TTR amyloid formation are summarized in Figure 2. Although some the available therapies are more efficient than others, it becomes increasingly evident that combination of different therapies may improve the therapeutic outcome. In this sense, it would be interesting to test TTR gene silencing therapies in combination with protein stabilizers or disruptors of pre-existing amyloid

\section{REFERENCES}

Ackermann, E. J., Guo, S., Benson, M. D., Booten, S., Freier, S., Hughes, S. G., et al. (2016). Suppressing transthyretin production in mice, monkeys and humans using 2nd-Generation antisense oligonucleotides. Amyloid 23, 148-157. doi: 10.1080/13506129.2016.1191458

Adams, D., Gonzalez-Duarte, A., O’Riordan, W. D., Yang, C. C., Ueda, M., Kristen, A. V., et al. (2018). Patisiran, an RNAi Therapeutic, for Hereditary Transthyretin Amyloidosis. N. Engl. J. Med. 379, 11-21. doi: 10.1056/NEJMoa1716153

Adams, D., Theaudin, M., Cauquil, C., Algalarrondo, V., and Slama, M. (2014). FAP neuropathy and emerging treatments. Curr. Neurol. Neurosci. Rep. 14:435. doi: 10.1007/s11910-013-0435-3

Alhamadsheh, M. M., Connelly, S., Cho, A., Reixach, N., Powers, E. T., Pan, D. W., et al. (2011). Potent kinetic stabilizers that prevent transthyretinmediated cardiomyocyte proteotoxicity. Sci. Transl. Med. 3:97ra81. doi: 10.1126/scitranslmed.3002473

Ando, E., Ando, Y., and Haraoka, K. (2001). Ocular amyloid involvement after liver transplantation for polyneuropathy. Ann. Intern. Med. 135, 931-932. doi: 10.7326/0003-4819-135-10-200111200-00026

Ando, E., Ando, Y., Okamura, R., Uchino, M., Ando, M., and Negi, A. (1997). Ocular manifestations of familial amyloidotic polyneuropathy type I: long-term follow up. Br. J. Ophthalmol. 81, 295-298. doi: 10.1136/bjo.81.4.295

Ando, Y., Nakamura, M., and Araki, S. (2005). Transthyretin-related familial amyloidotic polyneuropathy. Arch. Neurol. 62, 1057-1062. doi: 10.1001/archneur.62.7.1057

Andrade, C. (1952). A peculiar form of peripheral neuropathy; familiar atypical generalized amyloidosis with special involvement of the peripheral nerves. Brain 75, 408-427. doi: 10.1093/brain/75.3.408

Andrea, T. A., Cavalieri, R. R., Goldfine, I. D., and Jorgensen, E. C. (1980). Binding of thyroid hormones and analogues to the human plasma protein prealbumin. Biochemistry 19, 55-63. doi: 10.1021/bi0054 2a009

aus dem Siepen, F., Bauer, R., Aurich, M., Buss, S. J., Steen, H., Altland, K., et al. (2015). Green tea extract as a treatment for patients with wild-type deposits. It is also important to obtain more efficient and targeted therapies specific to organ and tissues with limited drug access as is the case of the eye and brain, that are particularly relevant in some forms of the disease. Moreover, it is crucial to continue with studies that can contribute to a better understanding of the mechanisms involved in the disease, in particular, TTR proteolysis, which has been mainly valued in the case of ATTR-CM and, also at the extracellular level involving either interactions with components of the extracellular matrix or with molecular and chemical chaperones acting as disease modulators.

Overall, detailed knowledge of the mechanisms of amyloid formation and the availability of different approaches allows directed and personalized interventions aiming higher specificity and efficacy of chosen therapeutic solutions.

\section{AUTHOR CONTRIBUTIONS}

FB, MS, and MA wrote and discussed the manuscript. All authors contributed to the article and approved the submitted version.

\section{FUNDING}

FB was supported by FCT- Fundação para a Ciência e Tecnologia/MEC - Ministério da Educação e Ciência with a Ph.D. fellowship (SFRH/BD/123674/2016).

transthyretin amyloidosis: an observational study. Drug Des. Devel. Ther. 9, 6319-6325. doi: 10.2147/DDDT.S96893

Barreiros, A. P., Post, F., Hoppe-Lotichius, M., Linke, R. P., Vahl, C. F., Schafers, H. J., et al. (2010). Liver transplantation and combined liver-heart transplantation in patients with familial amyloid polyneuropathy: a single-center experience. Liver Transpl. 16, 314-323. doi: 10.1002/lt.21996

Barroso, F. A., Judge, D. P., Ebede, B., Li, H., Stewart, M., Amass, L., et al. (2017). Long-term safety and efficacy of tafamidis for the treatment of hereditary transthyretin amyloid polyneuropathy: results up to 6 years. Amyloid 24, 194-204. doi: 10.1080/13506129.2017.1357545

Baures, P. W., Oza, V. B., Peterson, S. A., and Kelly, J. W. (1999). Synthesis and evaluation of inhibitors of transthyretin amyloid formation based on the non-steroidal anti-inflammatory drug, flufenamic acid. Bioorg. Med. Chem. 7, 1339-1347.

Benson, M. D. (2013). Liver transplantation and transthyretin amyloidosis. Muscle Nerve 47, 157-162. doi: 10.1002/mus.23521

Benson, M. D., Buxbaum, J. N., Eisenberg, D. S., Merlini, G., Saraiva, M. J. M., Sekijima, Y., et al. (2018a). Amyloid nomenclature 2018: recommendations by the International Society of Amyloidosis (ISA) nomenclature committee. Amyloid 25, 215-219. doi: 10.1080/13506129.2018.1549825

Benson, M. D., Waddington-Cruz, M., Berk, J. L., Polydefkis, M., Dyck, P. J., Wang, A. K., et al. (2018b). Inotersen treatment for patients with hereditary transthyretin amyloidosis. N. Engl. J. Med. 379, 22-31. doi: 10.1056/NEJMoa1716793

Bergstrom, J., Gustavsson, A., Hellman, U., Sletten, K., Murphy, C. L., Weiss, D. T., et al. (2005). Amyloid deposits in transthyretin-derived amyloidosis: cleaved transthyretin is associated with distinct amyloid morphology. J. Pathol. 206, 224-232. doi: 10.1002/path.1759

Berk, J. L., Suhr, O. B., Obici, L., Sekijima, Y., Zeldenrust, S. R., Yamashita, T., et al. (2013). Repurposing diflunisal for familial amyloid polyneuropathy: a randomized clinical trial. JAMA 310, 2658-2667. doi: 10.1001/jama.2013.283815

Blake, C. C., Geisow, M. J., Oatley, S. J., Rerat, B., and Rerat, C. (1978). Structure of prealbumin: secondary, tertiary and quaternary interactions determined by Fourier refinement at 1.8 A. J. Mol. Biol. 121, 339-356. 
Blake, C. C., Geisow, M. J., Swan, I. D., Rerat, C., and Rerat, B. (1974). Structure of human plasma prealbumin at 2-5 A resolution. A preliminary report on the polypeptide chain conformation, quaternary structure and thyroxine binding. $J$ Mol Biol 88, 1-12.

Bouma, B., Maas, C., Hazenberg, B. P., Lokhorst, H. M., and Gebbink, M. F. (2007). Increased plasmin-alpha2-antiplasmin levels indicate activation of the fibrinolytic system in systemic amyloidoses. J. Thromb Haemost. 5, 1139-1142. doi: 10.1111/j.1538-7836.2007.02457.x

Bulawa, C. E., Connelly, S., Devit, M., Wang, L., Weigel, C., Fleming, J. A., et al. (2012). Tafamidis, a potent and selective transthyretin kinetic stabilizer that inhibits the amyloid cascade. Proc Natl Acad Sci U S A 109, 9629-9634. doi: 10.1073/pnas.1121005109

Butler, J. S., Chan, A., Costelha, S., Fishman, S., Willoughby, J. L., Borland, T. D., et al. (2016). Preclinical evaluation of RNAi as a treatment for transthyretin-mediated amyloidosis. Amyloid 23, 109-118. doi: $10.3109 / 13506129.2016 .1160882$

Buxbaum, J. N., and Reixach, N. (2009). Transthyretin: the servant of many masters. Cell Mol. Life Sci. 66, 3095-3101. doi: 10.1007/s00018-009-0109-0

Buxbaum, J. N., and Ruberg, F. L. (2017). Transthyretin V122I (pV142I)* cardiac amyloidosis: an age-dependent autosomal dominant cardiomyopathy too common to be overlooked as a cause of significant heart disease in elderly African Americans. Genet. Med. 19, 733-742. doi: 10.1038/gim.2016.200

Cappelli, F., Martone, R., Taborchi, G., Morini, S., Bartolini, S., Angelotti, P., et al. (2018). Epigallocatechin-3-gallate tolerability and impact on survival in a cohort of patients with transthyretin-related cardiac amyloidosis. A single-center retrospective study. Intern. Emerg. Med. 13, 873-880. doi: $10.1007 /$ s11739-018-1887-x

Cardoso, I., Brito, M., and Saraiva, M. J. (2008). Extracellular matrix markers for disease progression and follow-up of therapies in familial amyloid polyneuropathy V30M TTR-related. Dis. Markers 25, 37-47. doi: $10.1155 / 2008 / 549872$

Cardoso, I., Goldsbury, C. S., Muller, S. A., Olivieri, V., Wirtz, S., Damas, A. M., et al. (2002). Transthyretin fibrillogenesis entails the assembly of monomers: a molecular model for in vitro assembled transthyretin amyloid-like fibrils. J. Mol. Biol. 317, 683-695. doi: 10.1006/jmbi.2002.5441

Cardoso, I., Martins, D., Ribeiro, T., Merlini, G., and Saraiva, M. J. (2010). Synergy of combined doxycycline/TUDCA treatment in lowering Transthyretin deposition and associated biomarkers: studies in FAP mouse models. J. Transl. Med. 8:74. doi: 10.1186/1479-5876-8-74

Cardoso, I., Merlini, G., and Saraiva, M. J. (2003). 4'-iodo-4'-deoxydoxorubicin and tetracyclines disrupt transthyretin amyloid fibrils in vitro producing noncytotoxic species: screening for TTR fibril disrupters. FASEB J. 17, 803-809. doi: 10.1096/fj.02-0764com

Cardoso, I., and Saraiva, M. J. (2006). Doxycycline disrupts transthyretin amyloid: evidence from studies in a FAP transgenic mice model. FASEB J. 20, 234-239. doi: 10.1096/fj.05-4509com

Chen, R., Chen, C. P., and Preston, J. E. (2016). Effects of transthyretin on thyroxine and beta-amyloid removal from cerebrospinal fluid in mice. Clin. Exp. Pharmacol. Physiol. 43, 844-850. doi: 10.1111/1440-1681.12598

Coelho, T., Adams, D., Silva, A., Lozeron, P., Hawkins, P. N., Mant, T., et al. (2013). Safety and efficacy of RNAi therapy for transthyretin amyloidosis. N. Engl. J. Med. 369, 819-829. doi: 10.1056/NEJMoa1208760

Coelho, T., Maia, L. F., Martins da Silva, A., Waddington Cruz, M., PlanteBordeneuve, V., Lozeron, P., et al. (2012). Tafamidis for transthyretin familial amyloid polyneuropathy: a randomized, controlled trial. Neurology 79, 785-792. doi: 10.1212/WNL.0b013e3182661ebl

Coelho, T., Merlini, G., Bulawa, C. E., Fleming, J. A., Judge, D. P., Kelly, J. W., et al. (2016). Mechanism of Action and Clinical Application of Tafamidis in Hereditary Transthyretin Amyloidosis. Neurol. Ther. 5, 1-25. doi: 10.1007/s40120-016-0040-x

Conceicao, I., Coelho, T., Rapezzi, C., Parman, Y., Obici, L., Galan, L., et al. (2019). Assessment of patients with hereditary transthyretin amyloidosis understanding the impact of management and disease progression. Amyloid 26, 103-111. doi: 10.1080/13506129.2019.1627312

Conceicao, I., Gonzalez-Duarte, A., Obici, L., Schmidt, H. H., Simoneau, D., Ong, M. L., et al. (2016). "Red-flag" symptom clusters in transthyretin familial amyloid polyneuropathy. J. Peripher Nerv. Syst. 21, 5-9. doi: 10.1111/jns.12153
Conejos-Sanchez, I., Cardoso, I., Oteo-Vives, M., Romero-Sanz, E., Paul, A., Sauri, A. R., et al. (2015). Polymer-doxycycline conjugates as fibril disrupters: an approach towards the treatment of a rare amyloidotic disease. J. Control Release 198, 80-90. doi: 10.1016/j.jconrel.2014.12.003

Connors, L. H., Lim, A., Prokaeva, T., Roskens, V. A., and Costello, C. E. (2003). Tabulation of human transthyretin (TTR) variants, 2003. Amyloid 10, 160-184. doi: 10.3109/13506120308998998

Constantinescu, P., Brown, R. A., Wyatt, A. R., Ranson, M., and Wilson, M. R. (2017). Amorphous protein aggregates stimulate plasminogen activation, leading to release of cytotoxic fragments that are clients for extracellular chaperones. J. Biol. Chem. 292, 14425-14437. doi: 10.1074/jbc.M117.786657

Corazza, A., Verona, G., Waudby, C. A., Mangione, P. P., Bingham, R., Uings, I., et al. (2019). Binding of monovalent and bivalent ligands by transthyretin causes different short- and long-distance conformational changes. J. Med. Chem. 62, 8274-8283. doi: 10.1021/acs.jmedchem.9b01037

Costa, R., Ferreira-da-Silva, F., Saraiva, M. J., and Cardoso, I. (2008). Transthyretin protects against A-beta peptide toxicity by proteolytic cleavage of the peptide: a mechanism sensitive to the Kunitz protease inhibitor. PLOS ONE 3:e2899. doi: 10.1371/journal.pone.0002899

da Costa, G., Ribeiro-Silva, C., Ribeiro, R., Gilberto, S., Gomes, R. A., Ferreira, A., et al. (2015). Transthyretin amyloidosis: chaperone concentration changes and increased proteolysis in the pathway to disease. PLOS ONE 10:e0125392. doi: 10.1371/journal.pone.0125392

DeLano, W. (2005). The PyMOL Molecular Graphics System. San Carlos: DeLano Scientific.

Dessi, A., Peluso, P., Dallocchio, R., Weiss, R., Andreotti, G., Allocca, M., et al. (2020). Rational design, synthesis, characterization and evaluation of iodinated 4,4'-bipyridines as new transthyretin fibrillogenesis inhibitors. Molecules 25:2213. doi: 10.3390/molecules25092213

Donnelly, J. P., and Hanna, M. (2017). Cardiac amyloidosis: an update on diagnosis and treatment. Cleve Clin. J. Med. 84 (12 Suppl. 3), 12-26. doi: $10.3949 /$ ccjm.84.s3.02

Ericzon, B. G., Wilczek, H. E., Larsson, M., Wijayatunga, P., Stangou, A., Pena, J. R., et al. (2015). Liver transplantation for hereditary transthyretin amyloidosis: after 20 years still the best therapeutic alternative? Transplantation 99, 1847-1854. doi: 10.1097/TP.0000000000000574

Falk, R. H., and Dubrey, S. W. (2010). Amyloid heart disease. Prog. Cardiovasc. Dis. 52, 347-361. doi: 10.1016/j.pcad.2009.11.007

Ferreira, N., Cardoso, I., Domingues, M. R., Vitorino, R., Bastos, M., Bai, G., et al. (2009). Binding of epigallocatechin-3-gallate to transthyretin modulates its amyloidogenicity. FEBS Lett. 583, 3569-3576. doi: 10.1016/j.febslet.2009.10.062

Ferreira, N., Goncalves, N. P., Saraiva, M. J., and Almeida, M. R. (2016). Curcumin: a multi-target disease-modifying agent for late-stage transthyretin amyloidosis. Sci. Rep. 6:26623. doi: 10.1038/srep26623

Ferreira, N., Pereira-Henriques, A., Attar, A., Klarner, F. G., Schrader, T., Bitan, G., et al. (2014). Molecular tweezers targeting transthyretin amyloidosis. Neurotherapeutics 11, 450-461. doi: 10.1007/s13311-013-0256-8

Ferreira, N., Santos, S. A., Domingues, M. R., Saraiva, M. J., and Almeida, M. R. (2013). Dietary curcumin counteracts extracellular transthyretin deposition: insights on the mechanism of amyloid inhibition. Biochim. Biophys. Acta 1832, 39-45. doi: 10.1016/j.bbadis.2012.10.007

Ferreira, N., Saraiva, M. J., and Almeida, M. R. (2011). Natural polyphenols inhibit different steps of the process of transthyretin (TTR) amyloid fibril formation. FEBS Lett. 585, 2424-2430. doi: 10.1016/j.febslet.2011.06.030

Ferreira, N., Saraiva, M. J., and Almeida, M. R. (2012). Epigallocatechin3-gallate as a potential therapeutic drug for TTR-related amyloidosis: "in vivo" evidence from FAP mice models. PLoS ONE 7:e29933. doi: 10.1371/journal.pone.0029933

Ferreira, N., Saraiva, M. J., and Almeida, M. R. (2019). Uncovering the neuroprotective mechanisms of curcumin on transthyretin amyloidosis. Int. J. Mol. Sci. 20:1287. doi: 10.3390/ijms20061287

Folli, C., Favilla, R., and Berni, R. (2010). The interaction between retinol-binding protein and transthyretin analyzed by fluorescence anisotropy. Methods Mol. Biol. 652, 189-207. doi: 10.1007/978-1-60327-325-1_11

French, K., Yerbury, J. J., and Wilson, M. R. (2008). Protease activation of alpha2macroglobulin modulates a chaperone-like action with broad specificity. Biochemistry 47, 1176-1185. doi: 10.1021/bi701976f 
Gaetani, S., Bellovino, D., Apreda, M., and Devirgiliis, C. (2002). Hepatic synthesis, maturation and complex formation between retinol-binding protein and transthyretin. Clin. Chem. Lab. Med. 40, 1211-1220. doi: 10.1515/CCLM.2002.211

Galant, N. J., Bugyei-Twum, A., Rakhit, R., Walsh, P., Sharpe, S., Arslan, P. E., et al. (2016). Substoichiometric inhibition of transthyretin misfolding by immunetargeting sparsely populated misfolding intermediates: a potential diagnostic and therapeutic for TTR amyloidoses. Sci. Rep. 6:25080. doi: 10.1038/srep25080

Galant, N. J., Westermark, P., Higaki, J. N., and Chakrabartty, A. (2017). Transthyretin amyloidosis: an under-recognized neuropathy and cardiomyopathy. Clin. Sci. 131, 395-409. doi: 10.1042/CS20160413

Gamez, J., Salvado, M., Reig, N., Sune, P., Casasnovas, C., Rojas-Garcia, R., et al. (2019). Transthyretin stabilization activity of the catechol-O-methyltransferase inhibitor tolcapone (SOM0226) in hereditary ATTR amyloidosis patients and asymptomatic carriers: proof-of-concept study(). Amyloid 26, 74-84. doi: 10.1080/13506129.2019.1597702

Gertz, M. A., Mauermann, M. L., Grogan, M., and Coelho, T. (2019). Advances in the treatment of hereditary transthyretin amyloidosis: a review. Brain Behav. 9:e01371. doi: 10.1002/brb3.1371

Gomes, J. R., Nogueira, R. S., Vieira, M., Santos, S. D., Ferraz-Nogueira, J. P., Relvas, J. B., et al. (2016). Transthyretin provides trophic support via megalin by promoting neurite outgrowth and neuroprotection in cerebral ischemia. Cell Death Differ 23, 1749-1764. doi: 10.1038/cdd.2016.64

Gomes, J. R., Sarkany, Z., Teixeira, A., Nogueira, R., Cabrito, I., Soares, H., et al. (2019). Anti-TTR nanobodies allow the identification of TTR neuritogenic epitope associated with TTR-megalin neurotrophic activities. ACS Chem. Neurosci. 10, 704-715. doi: 10.1021/acschemneuro.8b00502

Goto, T., Yamashita, T., Ueda, M., Ohshima, S., Yoneyama, K., Nakamura, M., et al. (2006). Iatrogenic amyloid neuropathy in a Japanese patient after sequential liver transplantation. Am. J. Transplant 6, 2512-2515. doi: 10.1111/j.1600-6143.2006.01484.x

Greene, M. J., Klimtchuk, E. S., Seldin, D. C., Berk, J. L., and Connors, L. H. (2015). Cooperative stabilization of transthyretin by clusterin and diflunisal. Biochemistry 54, 268-278. doi: 10.1021/bi5011249

Greene, M. J., Sam, F., Soo Hoo, P. T., Patel, R. S., Seldin, D. C., and Connors, L. H. (2011). Evidence for a functional role of the molecular chaperone clusterin in amyloidotic cardiomyopathy. Am. J. Pathol. 178, 61-68. doi: 10.1016/j.ajpath.2010.11.015

Gustafsson, S., Ihse, E., Henein, M. Y., Westermark, P., Lindqvist, P., and Suhr, O. B. (2012). Amyloid fibril composition as a predictor of development of cardiomyopathy after liver transplantation for hereditary transthyretin amyloidosis. Transplantation 93, 1017-1023. doi: 10.1097/TP.0b013e31824b3749

Habtemariam, B. A., Karsten, V., Attarwala, H., Goel, V., Melch, M., Clausen, V. A., et al. (2020). Single-dose pharmacokinetics and pharmacodynamics of transthyretin targeting $n$-acetylgalactosamine-small interfering ribonucleic acid conjugate, vutrisiran, in healthy subjects. Clin. Pharmacol. Ther. doi: 10.1002/cpt.1974. [Epub ahead of print].

$\mathrm{Hu}, \mathrm{S}$., Loo, J. A., and Wong, D. T. (2006). Human body fluid proteome analysis. Proteomics 6, 6326-6353. doi: 10.1002/pmic.200600284

Ihse, E., Rapezzi, C., Merlini, G., Benson, M. D., Ando, Y., Suhr, O. B., et al. (2013). Amyloid fibrils containing fragmented ATTR may be the standard fibril composition in ATTR amyloidosis. Amyloid 20, 142-150. doi: 10.3109/13506129.2013.797890

Ihse, E., Suhr, O. B., Hellman, U., and Westermark, P. (2011). Variation in amount of wild-type transthyretin in different fibril and tissue types in ATTR amyloidosis. J. Mol. Med. 89, 171-180. doi: 10.1007/s00109-010-0695-1

Ihse, E., Ybo, A., Suhr, O., Lindqvist, P., Backman, C., and Westermark, P. (2008). Amyloid fibril composition is related to the phenotype of hereditary transthyretin V30M amyloidosis. J. Pathol. 216, 253-261. doi: $10.1002 /$ path.2411

Jacobson, D. R., Pastore, R. D., Yaghoubian, R., Kane, I., Gallo, G., Buck, F. S., et al. (1997). Variant-sequence transthyretin (isoleucine 122) in late-onset cardiac amyloidosis in black Americans. N. Engl. J. Med. 336, 466-473. doi: 10.1056/NEJM199702133360703

Judge, D. P., Heitner, S. B., Falk, R. H., Maurer, M. S., Shah, S. J., Witteles, R. M., et al. (2019). Transthyretin Stabilization by AG10 in Symptomatic
Transthyretin Amyloid Cardiomyopathy. J. Am. Coll. Cardiol 74, 285-295. doi: 10.1016/j.jacc.2019.03.012

Kanda, Y., Goodman, D. S., Canfield, R. E., and Morgan, F. J. (1974). The amino acid sequence of human plasma prealbumin. J. Biol. Chem 249, 6796-6805.

Kato-Motozaki, Y., Ono, K., Shima, K., Morinaga, A., Machiya, T., Nozaki, I., et al. (2008). Epidemiology of familial amyloid polyneuropathy in Japan: Identification of a novel endemic focus. J. Neurol. Sci. 270, 133-140. doi: 10.1016/j.jns.2008.02.019

Koike, H., and Katsuno, M. (2020). Transthyretin amyloidosis: update on the clinical spectrum, pathogenesis, and disease-modifying therapies. Neurol Ther. 1-17. doi: 10.1007/s40120-020-00210-7. [Epub ahead of print].

Koike, H., Kiuchi, T., Iijima, M., Ueda, M., Ando, Y., Morozumi, S., et al. (2011). Systemic but asymptomatic transthyretin amyloidosis 8 years after domino liver transplantation. J. Neurol. Neurosurg. Psychiatr. 82, 1287-1290. doi: 10.1136/jnnp.2010.218958

Kolstoe, S. E., Mangione, P. P., Bellotti, V., Taylor, G. W., Tennent, G. A., Deroo, S., et al. (2010). Trapping of palindromic ligands within native transthyretin prevents amyloid formation. Proc. Natl. Acad. Sci. U.S.A. 107, 20483-20488. doi: 10.1073/pnas.1008255107

Kristen, A. V., Lehrke, S., Buss, S., Mereles, D., Steen, H., Ehlermann, P., et al. (2012). Green tea halts progression of cardiac transthyretin amyloidosis: an observational report. Clin. Res. Cardiol. 101, 805-813. doi: 10.1007/s00392-012-0463-z

Lee, K. W., Lee, D. H., Son, H., Kim, Y. S., Park, J. Y., Roh, G. S., et al. (2009). Clusterin regulates transthyretin amyloidosis. Biochem. Biophys. Res. Commun. 388, 256-260. doi: 10.1016/j.bbrc.2009.07.166

Lewis, W. D., Skinner, M., Simms, R. W., Jones, L. A., Cohen, A. S., and Jenkins, R. L. (1994). Orthotopic liver transplantation for familial amyloidotic polyneuropathy. Clin Transplant 8(2 Pt 1), 107-110.

Liepnieks, J. J., Wilson, D. L., and Benson, M. D. (2006). Biochemical characterization of vitreous and cardiac amyloid in Ile84Ser transthyretin amyloidosis. Amyloid 13, 170-177. doi: 10.1080/135061206008 77003

Liz, M. A., Faro, C. J., Saraiva, M. J., and Sousa, M. M. (2004). Transthyretin, a new cryptic protease. J. Biol. Chem 279, 21431-21438. doi: 10.1074/jbc.M402212200

Liz, M. A., Fleming, C. E., Nunes, A. F., Almeida, M. R., Mar, F. M., Choe, Y., et al. (2009). Substrate specificity of transthyretin: identification of natural substrates in the nervous system. Biochem. J. 419, 467-474. doi: 10.1042/BJ20082090

Liz, M. A., Mar, F. M., Franquinho, F., and Sousa, M. M. (2010). Aboard transthyretin: From transport to cleavage. IUBMB Life 62, 429-435. doi: $10.1002 /$ iub. 340

Macedo, A. V. S., Schwartzmann, P. V., de Gusmao, B. M., Melo, M. D. T., and Coelho-Filho, O. R. (2020). Advances in the Treatment of Cardiac Amyloidosis. Curr. Treat. Options Oncol. 21:36. doi: 10.1007/s11864-020-00738-8

Macedo, B., Batista, A. R., Ferreira, N., Almeida, M. R., and Saraiva, M. J. (2008). Anti-apoptotic treatment reduces transthyretin deposition in a transgenic mouse model of Familial Amyloidotic Polyneuropathy. Biochim. Biophys. Acta 1782, 517-522. doi: 10.1016/j.bbadis.2008.05.005

Magalhaes, J., and Saraiva, M. J. (2011). Clusterin overexpression and its possible protective role in transthyretin deposition in familial amyloidotic polyneuropathy. J. Neuropathol. Exp. Neurol. 70, 1097-1106. doi: 10.1097/NEN.0b013e31823a44f4

Maia, L. F., Magalhaes, R., Freitas, J., Taipa, R., Pires, M. M., Osorio, H., et al. (2015). CNS involvement in V30M transthyretin amyloidosis: clinical, neuropathological and biochemical findings. J. Neurol. Neurosurg. Psychiatr. 86, 159-167. doi: 10.1136/jnnp-2014-308107

Mangione, P. P., Porcari, R., Gillmore, J. D., Pucci, P., Monti, M., Porcari, M., et al. (2014). Proteolytic cleavage of Ser52Pro variant transthyretin triggers its amyloid fibrillogenesis. Proc. Natl. Acad. Sci. U.S.A. 111, 1539-1544. doi: $10.1073 /$ pnas.1317488111

Mangione, P. P., Verona, G., Corazza, A., Marcoux, J., Canetti, D., Giorgetti, S., et al. (2018). Plasminogen activation triggers transthyretin amyloidogenesis in vitro. J. Biol. Chem. 293, 14192-14199. doi: 10.1074/jbc.RA118.003990

Marcoux, J., Mangione, P. P., Porcari, R., Degiacomi, M. T., Verona, G., Taylor, G. W., et al. (2015). A novel mechano-enzymatic cleavage mechanism underlies transthyretin amyloidogenesis. EMBO Mol. Med. 7, 1337-1349. doi: 10.15252/emmm.201505357 
Matsubara, E., Soto, C., Governale, S., Frangione, B., and Ghiso, J. (1996). Apolipoprotein J and Alzheimer's amyloid beta solubility. Biochem. J. 316 (Pt 2), 671-679.

Maurer, M. S., Bokhari, S., Damy, T., Dorbala, S., Drachman, B. M., Fontana, M., et al. (2019). Expert consensus recommendations for the suspicion and diagnosis of transthyretin cardiac amyloidosis. Circ. Heart Fail. 12:e006075. doi: 10.1161/CIRCHEARTFAILURE.119.006075

Maurer, M. S., Hanna, M., Grogan, M., Dispenzieri, A., Witteles, R., Drachman, B., et al. (2016). Genotype and phenotype of transthyretin cardiac Amyloidosis: THAOS (transthyretin amyloid outcome survey). J. Am. Coll. Cardiol. 68, 161-172. doi: 10.1016/j.jacc.2016.03.596

Maurer, M. S., Schwartz, J. H., Gundapaneni, B., Elliott, P. M., Merlini, G., Waddington-Cruz, M., et al. (2018). Tafamidis treatment for patients with transthyretin amyloid cardiomyopathy. N. Engl. J. Med. 379, 1007-1016. doi: 10.1056/NEJMoa1805689

McCutchen, S. L., Colon, W., and Kelly, J. W. (1993). Transthyretin mutation Leu55-Pro significantly alters tetramer stability and increases amyloidogenicity. Biochemistry 32, 12119-12127. doi: 10.1021/bi00096a024

Merlini, G., Ascari, E., Amboldi, N., Bellotti, V., Arbustini, E., Perfetti, V., et al. (1995). Interaction of the anthracycline 4'-iodo-4'-deoxydoxorubicin with amyloid fibrils: inhibition of amyloidogenesis. Proc. Natl. Acad. Sci. U.S.A. 92, 2959-2963. doi: 10.1073/pnas.92.7.2959

Miller, M., Pal, A., Albusairi, W., Joo, H., Pappas, B., Haque Tuhin, M. T., et al. (2018). Enthalpy-driven stabilization of transthyretin by AG10 mimics a naturally occurring genetic variant that protects from transthyretin amyloidosis. J. Med. Chem. 61, 7862-7876. doi: 10.1021/acs.jmedchem.8b00817

Miller, S. R., Sekijima, Y., and Kelly, J. W. (2004). Native state stabilization by NSAIDs inhibits transthyretin amyloidogenesis from the most common familial disease variants. Lab. Invest. 84, 545-552. doi: 10.1038/labinvest.3700059

Miroy, G. J., Lai, Z., Lashuel, H. A., Peterson, S. A., Strang, C., and Kelly, J. W. (1996). Inhibiting transthyretin amyloid fibril formation via protein stabilization. Proc. Natl. Acad. Sci. U.S.A. 93, 15051-15056.

Mukherjee, D., Nissen, S. E., and Topol, E. J. (2001). Risk of cardiovascular events associated with selective COX-2 inhibitors. JAMA 286, 954-959. doi: 10.1001/jama.286.8.954

Mumford, A. D., O’Donnell, J., Gillmore, J. D., Manning, R. A., Hawkins, P. N., and Laffan, M. (2000). Bleeding symptoms and coagulation abnormalities in 337 patients with AL-amyloidosis. Br. J. Haematol. 110, 454-460. doi: 10.1046/j.1365-2141.2000.02183.x

Munar-Ques, M., Salva-Ladaria, L., Mulet-Perera, P., Sole, M., Lopez-Andreu, F. R., and Saraiva, M. J. (2000). Vitreous amyloidosis after liver transplantation in patients with familial amyloid polyneuropathy: ocular synthesis of mutant transthyretin. Amyloid 7, 266-269. doi: 10.3109/13506120009146440

Murakami, T., Ohsawa, Y., Zhenghua, L., Yamamura, K., and Sunada, Y. (2010). The transthyretin gene is expressed in Schwann cells of peripheral nerves. Brain Res. 1348, 222-225. doi: 10.1016/j.brainres.2010.06.017

Obici, L., and Merlini, G. (2014). An overview of drugs currently under investigation for the treatment of transthyretin-related hereditary amyloidosis. Expert Opin. Investig. Drugs 23, 1239-1251. doi: 10.1517/13543784.2014.922541

Okamoto, S., Zhao, Y., Lindqvist, P., Backman, C., Ericzon, B. G., Wijayatunga, P., et al. (2011). Development of cardiomyopathy after liver transplantation in Swedish hereditary transthyretin amyloidosis (ATTR) patients. Amyloid 18, 200-205. doi: 10.3109/13506129.2011.61 5872

Ortore, G., and Martinelli, A. (2012). Computational Studies on Transthyretin. Curr. Med. Chem. 19, 2380-2387. doi: 10.2174/0929867128002 69344

Patel, K. S., and Hawkins, P. N. (2015). Cardiac amyloidosis: where are we today? J. Intern. Med. 278, 126-144. doi: 10.1111/joim.12383

Penchala, S. C., Connelly, S., Wang, Y., Park, M. S., Zhao, L., Baranczak, A., et al. (2013). AG10 inhibits amyloidogenesis and cellular toxicity of the familial amyloid cardiomyopathy-associated V122I transthyretin. Proc. Natl. Acad. Sci. U.S.A. 110, 9992-9997. doi: 10.1073/pnas.1300761110

Pinheiro, F., Varejao, N., Esperante, S., Santos, J., Velazquez-Campoy, A., Reverter, D., et al. (2020). Tolcapone, a potent aggregation inhibitor for the treatment of familial leptomeningeal amyloidosis. FEBS J. doi: 10.1111/febs.15339. [Epub ahead of print].

Pitkanen, P., Westermark, P., and Cornwell, G. G. III. (1984). Senile systemic amyloidosis. Am. J. Pathol. 117, 391-399.

Plante-Bordeneuve, V., and Said, G. (2011). Familial amyloid polyneuropathy. Lancet Neurol. 10, 1086-1097. doi: 10.1016/S1474-4422(11)70246-0

Poon, S., Easterbrook-Smith, S. B., Rybchyn, M. S., Carver, J. A., and Wilson, M. R. (2000). Clusterin is an ATP-independent chaperone with very broad substrate specificity that stabilizes stressed proteins in a folding-competent state. Biochemistry 39, 15953-15960. doi: 10.1021/bi002189x

Quarta, C. C., Buxbaum, J. N., Shah, A. M., Falk, R. H., Claggett, B., Kitzman, D. W., et al. (2015). The amyloidogenic V122I transthyretin variant in elderly black Americans. N. Engl. J. Med. 372, 21-29. doi: 10.1056/NEJMoa 1404852

Quintas, A., Saraiva, M. J., and Brito, R. M. (1999). The tetrameric protein transthyretin dissociates to a non-native monomer in solution. A novel model for amyloidogenesis. J. Biol. Chem. 274, 32943-32949. doi: $10.1074 /$ jbc. 274.46 .32943

Quintas, A., Vaz, D. C., Cardoso, I., Saraiva, M. J., and Brito, R. M. (2001). Tetramer dissociation and monomer partial unfolding precedes protofibril formation in amyloidogenic transthyretin variants. J. Biol. Chem. 276, 27207-27213. doi: 10.1074/jbc.M101024200

Raghu, P., and Sivakumar, B. (2004). Interactions amongst plasma retinolbinding protein, transthyretin and their ligands: implications in vitamin A homeostasis and transthyretin amyloidosis. Biochim. Biophys. Acta 1703, 1-9. doi: 10.1016/j.bbapap.2004.09.023

Rapezzi, C., Lorenzini, M., Longhi, S., Milandri, A., Gagliardi, C., Bartolomei, I., et al. (2015). Cardiac amyloidosis: the great pretender. Heart Fail. Rev. 20, 117-124. doi: 10.1007/s10741-015-9480-0

Rapezzi, C., Quarta, C. C., Riva, L., Longhi, S., Gallelli, I., Lorenzini, M., et al. (2010). Transthyretin-related amyloidoses and the heart: a clinical overview. Nat. Rev. Cardiol. 7, 398-408. doi: 10.1038/nrcardio.2010.67

Refetoff, S. (2000). "Thyroid hormone serum transport proteins," in Endotext [Internet], eds K. R. Feingold, B. Anawalt, A. Boyce, et al. South Dartmouth, MA: MDText.com, Inc.

Richardson, S. J. (2009). Evolutionary changes to transthyretin: evolution of transthyretin biosynthesis. FEBS J. 276, 5342-5356. doi: 10.1111/j.1742-4658.2009.07244.x

Saelices, L., Chung, K., Lee, J. H., Cohn, W., Whitelegge, J. P., Benson, M. D., et al. (2018). Amyloid seeding of transthyretin by ex vivo cardiac fibrils and its inhibition. Proc. Natl. Acad. Sci. U.S.A. 115, E6741-E6750. doi: $10.1073 /$ pnas. 1805131115

Sant'Anna, R., Gallego, P., Robinson, L. Z., Pereira-Henriques, A., Ferreira, N. Pinheiro, F., et al. (2016). Repositioning tolcapone as a potent inhibitor of transthyretin amyloidogenesis and associated cellular toxicity. Nat. Commun. 7:10787. doi: $10.1038 /$ ncomms 10787

Santos, S. D., Lambertsen, K. L., Clausen, B. H., Akinc, A., Alvarez R., Finsen, B., et al. (2010). CSF transthyretin neuroprotection in a mouse model of brain ischemia. J. Neurochem. 115, 1434-1444. doi: 10.1111/j.1471-4159.2010.07047.x

Saraiva, M. J. (1995). Transthyretin mutations in health and disease. Hum. Mutat. 5, 191-196. doi: 10.1002/humu.1380050302

Saraiva, M. J., Birken, S., Costa, P. P., and Goodman, D. S. (1984). Amyloid fibril protein in familial amyloidotic polyneuropathy, Portuguese type. Definition of molecular abnormality in transthyretin (prealbumin). J. Clin. Invest. 74, 104-119. doi: 10.1172/JCI111390

Schormann, N., Murrell, J. R., and Benson, M. D. (1998). Tertiary structures of amyloidogenic and non-amyloidogenic transthyretin variants: new model for amyloid fibril formation. Amyloid 5, 175-187.

Sekijima, Y. (2015). Transthyretin (ATTR) amyloidosis: clinical spectrum, molecular pathogenesis and disease-modifying treatments. J. Neurol. Neurosurg. Psychiatr. 86, 1036-1043. doi: 10.1136/jnnp-2014-308724

Sekijima, Y., Tojo, K., Morita, H., Koyama, J., and Ikeda, S. (2015). Safety and efficacy of long-term diflunisal administration in hereditary transthyretin (ATTR) amyloidosis. Amyloid 22, 79-83. doi: 10.3109/13506129.2014.997872

Sekijima, Y., Yazaki, M., Oguchi, K., Ezawa, N., Yoshinaga, T., Yamada, M., et al. (2016). Cerebral amyloid angiopathy in posttransplant 
patients with hereditary ATTR amyloidosis. Neurology 87, 773-781. doi: 10.1212/WNL.0000000000003001

Sinha, S., Lopes, D. H., Du, Z., Pang, E. S., Shanmugam, A., Lomakin, A., et al. (2011). Lysine-specific molecular tweezers are broad-spectrum inhibitors of assembly and toxicity of amyloid proteins. J. Am. Chem. Soc. 133, 16958-16969. doi: $10.1021 /$ ja206279b

Sipe, J. D., Benson, M. D., Buxbaum, J. N., Ikeda, S. I., Merlini, G., Saraiva, M. J., et al. (2016). Amyloid fibril proteins and amyloidosis: chemical identification and clinical classification International Society of Amyloidosis 2016 Nomenclature Guidelines. Amyloid 23, 209-213. doi: 10.1080/13506129.2016.1257986

Sousa, A., Andersson, R., Drugge, U., Holmgren, G., and Sandgren, O. (1993). Familial amyloidotic polyneuropathy in Sweden: geographical distribution, age of onset, and prevalence. Hum. Hered. 43, 288-294. doi: 10.1159/000154146

Sousa, A., Coelho, T., Barros, J., and Sequeiros, J. (1995). Genetic epidemiology of familial amyloidotic polyneuropathy (FAP)-type I in Povoa do Varzim and Vila do Conde (north of Portugal). Am. J. Med. Genet. 60, 512-521. doi: 10.1002/ajmg.1320600606

Sousa, M. M., Berglund, L., and Saraiva, M. J. (2000). Transthyretin in high density lipoproteins: association with apolipoprotein A-I. J. Lipid Res 41, 58-65.

Stangou, A. J., Heaton, N. D., and Hawkins, P. N. (2005). Transmission of systemic transthyretin amyloidosis by means of domino liver transplantation. N. Engl. J. Med. 352:2356. doi: 10.1056/NEJM200506023522219

Suhr, O. B., Lundgren, E., and Westermark, P. (2017). One mutation, two distinct disease variants: unravelling the impact of transthyretin amyloid fibril composition. J. Intern. Med. 281, 337-347. doi: 10.1111/joim.12585

Suhr, O. B., Svendsen, I. H., Andersson, R., Danielsson, A., Holmgren, G., and Ranlov, P. J. (2003). Hereditary transthyretin amyloidosis from a Scandinavian perspective. J. Intern. Med. 254, 225-235. doi: 10.1046/j.1365-2796.2003.01173.x

Takahashi, R., Ono, K., Shibata, S., Nakamura, K., Komatsu, J., Ikeda, Y., et al. (2014). Efficacy of diflunisal on autonomic dysfunction of late-onset familial amyloid polyneuropathy (TTR Val30Met) in a Japanese endemic area. J. Neurol. Sci. 345, 231-235. doi: 10.1016/j.jns.2014.07.017

Teixeira, C. A., Almeida Mdo, R., and Saraiva, M. J. (2016). Impairment of autophagy by TTR V30M aggregates: in vivo reversal by TUDCA and curcumin. Clin. Sci. 130, 1665-1675. doi: 10.1042/CS20160075

Tojo, K., Sekijima, Y., Kelly, J. W., and Ikeda, S. (2006). Diflunisal stabilizes familial amyloid polyneuropathy-associated transthyretin variant tetramers in serum against dissociation required for amyloidogenesis. Neurosci. Res. 56, 441-449. doi: 10.1016/j.neures.2006.08.014

Torres-Arancivia, C., and Connors, L. H. (2019). Effect of diflunisal on clusterin levels in ATTRwt amyloidosis. Amyloid 26, 49-50. doi: 10.1080/13506129.2019.1582515

Tucker, H. M., Kihiko-Ehmann, M., Wright, S., Rydel, R. E., and Estus, S. (2000). Tissue plasminogen activator requires plasminogen to modulate amyloid-beta neurotoxicity and deposition. J. Neurochem. 75, 2172-2177. doi: 10.1046/j.1471-4159.2000.0752172.x

Uchiba, M., Imamura, T., Hata, H., Tatetsu, H., Yonemura, Y., Ueda, M., et al. (2009). Excessive fibrinolysis in AL-amyloidosis is induced by urokinae-type plasminogen activator from bone marrow plasma cells. Amyloid 16, 89-93. doi: 10.1080/13506120902879269

van Bennekum, A. M., Wei, S., Gamble, M. V., Vogel, S., Piantedosi, R., Gottesman, M., et al. (2001). Biochemical basis for depressed serum retinol levels in transthyretin-deficient mice. J. Biol. Chem. 276, 1107-1113. doi: 10.1074/jbc.M008091200

Verona, G., Mangione, P. P., Raimondi, S., Giorgetti, S., Faravelli, G., Porcari, R., et al. (2017). Inhibition of the mechano-enzymatic amyloidogenesis of transthyretin: role of ligand affinity, binding cooperativity and occupancy of the inner channel. Sci. Rep. 7:182. doi: 10.1038/s41598-01700338-x

Vieira, M., and Saraiva, M. J. (2014). Transthyretin: a multifaceted protein. Biomol. Concepts 5, 45-54. doi: 10.1515/bmc-2013-0038

Wei, S., Episkopou, V., Piantedosi, R., Maeda, S., Shimada, K., Gottesman, M. E., et al. (1995). Studies on the metabolism of retinol and retinol-binding protein in transthyretin-deficient mice produced by homologous recombination. J. Biol. Chem 270, 866-870. doi: 10.1074/jbc.270.2.866

Westermark, P., Bergstrom, J., Solomon, A., Murphy, C., and Sletten, K. (2003). Transthyretin-derived senile systemic amyloidosis: clinicopathologic and structural considerations. Amyloid 10 Suppl 1, 48-54.

Westermark, P., Sletten, K., Johansson, B., and Cornwell, G. G. III. (1990). Fibril in senile systemic amyloidosis is derived from normal transthyretin. Proc. Natl. Acad. Sci. U.S.A. 87, 2843-2845.

Wojtczak, A., Cody, V., Luft, J. R., and Pangborn, W. (1996). Structures of human transthyretin complexed with thyroxine at $2.0 \mathrm{~A}$ resolution and 3',5'-dinitro-N-acetyl-L-thyronine at $2.2 \mathrm{~A}$ resolution. Acta Crystallogr D Biol Crystallogr 52(Pt 4), 758-765. doi: 10.1107/S09074449960 03046

Wyatt, A., Yerbury, J., Poon, S., Dabbs, R., and Wilson, M. (2009). Chapter 6: The chaperone action of Clusterin and its putative role in quality control of extracellular protein folding. Adv. Cancer Res. 104, 89-114. doi: 10.1016/S0065-230X(09)04006-8

Wyatt, A. R., Yerbury, J. J., Ecroyd, H., and Wilson, M. R. (2013). Extracellular chaperones and proteostasis. Annu. Rev. Biochem. 82, 295-322. doi: 10.1146/annurev-biochem-072711-163904

Yerbury, J. J., Poon, S., Meehan, S., Thompson, B., Kumita, J. R., Dobson, C. M., et al. (2007). The extracellular chaperone clusterin influences amyloid formation and toxicity by interacting with prefibrillar structures. FASEB J. 21, 2312-2322. doi: 10.1096/fj.06-7986com

Yerbury, J. J., Rybchyn, M. S., Easterbrook-Smith, S. B., Henriques, C., and Wilson, M. R. (2005). The acute phase protein haptoglobin is a mammalian extracellular chaperone with an action similar to clusterin. Biochemistry 44, 10914-10925. doi: 10.1021/bi050764x

Yokoyama, T., Mizuguchi, M., Nabeshima, Y., Kusaka, K., Yamada, T., Hosoya, T., et al. (2012). Hydrogen-bond network and pH sensitivity in transthyretin: Neutron crystal structure of human transthyretin. J. Struct. Biol. 177, 283-290. doi: 10.1016/j.jsb.2011.12.022

Zhao, L. J., and Lei, M. (2014). Computational Chemical Studies on Transthyretin. Progress Chem. 26, 193-202. doi: 10.7536/PC1 30612

Zhou, S., Cheng, J., Yang, T., Ma, M., Zhang, W., Yuan, S., et al. (2019). Exploration of the misfolding mechanism of transthyretin monomer: insights from hybrid-resolution simulations and markov state model analysis. Biomolecules 9:889. doi: 10.3390/biom9120889

Conflict of Interest: The authors declare that the research was conducted in the absence of any commercial or financial relationships that could be construed as a potential conflict of interest.

Copyright (c) 2020 Bezerra, Saraiva and Almeida. This is an open-access article distributed under the terms of the Creative Commons Attribution License (CC BY). The use, distribution or reproduction in other forums is permitted, provided the original author(s) and the copyright owner(s) are credited and that the original publication in this journal is cited, in accordance with accepted academic practice. No use, distribution or reproduction is permitted which does not comply with these terms. 